\title{
FUNCTIONAL ANALYTIC PROPERTIES OF EXTREMELY AMENABLE SEMIGROUPS
}

\author{
BY \\ EDMOND E. GRANIRER
}

Introduction. Let $S$ be a semigroup $m(S)$ the Banach space of all bounded real functions on $S$ with the norm $\|f\|=\sup \{|f(s)| ; s \in S\}$ and $m(S)^{*}$ the conjugate Banach space of $m(S) . \phi \in m(S)^{*}$ is a mean if $\phi \geqq 0$ (i.e. $\phi(f) \geqq 0$ if $f \geqq 0, f \in m(S)$ ) and $\phi(1)=1$ (1 stands also for the constant one function on $S$ ). The semigroup $S$ is left amenable (LA) if there exists a mean $\phi \in m(S)^{*}$ which is left invariant i.e. $\phi\left(f_{a}\right)=\phi(f)$ for all $a \in S, f \in m(S)$ (we define $f_{a}(s)=f(a s), f^{a}(s)=f(s a), l_{a} f=f_{a}$, $r_{a} f=f^{a}, L_{a}=l_{a}^{*}: m(S)^{*} \rightarrow m(S)^{*}, R_{a}=r_{a}^{*}$, for all $a, s \in S$ and $\left.f \in m(S)\right)$. The semigroup $S$ is extremely left amenable (ELA) if there is a left invariant mean $\phi$ on $m(S)$ which is multiplicative (i.e. such that $\phi(f g)=\phi(f) \phi(g)$ for all $f, g \in m(S))$. In anology one defines (extremely) right amenable semigroups (ERA) RA.

ELA semigroups have been studied at first by $\mathrm{T}$. Mitchell in [22] (under different terminology) and afterwards by this author in [15], [16]. They are known to be characterized by the following equivalent properties:

1. $S$ is ELA.

2. $S$ has the common fixed point property on compacta (i.e. for any compact Hausdorff space and any homomorphic representation $S^{\prime}$ of $S$ as a semigroup of continuous maps from $X$ to $X$ (under functional composition), there is some $x_{0} \in X$ such that $s^{\prime}\left(x_{0}\right)=x_{0}$ for all $s^{\prime} \in S^{\prime}$ (Mitchell [22]).

3. For any $a, b \in S$ there is some $c \in S$ with $a c=b c=c$ [16].

4. For any function $h$ of the ideal

$$
H=\left\{\sum_{1}^{n} f_{j}\left(g_{j}-l_{s_{j}} g_{j}\right) ; s_{j} \in S, f_{j}, g_{j} \in m(S), n=1,2, \ldots\right\}
$$

there is some $a \in S$ such that $h(a)=0$.

(There still may be some $h$ in the closure $\bar{H}$ of $H$ with $h(s) \neq 0$ for all $s \in S$ even though $S$ is ELA [16].)

5. For any $f \in m(S)$, the pointwise closure of the right orbit $\left\{r_{s} f ; s \in S\right\}$ of $f$ contains some constant function [16].

6. $H$ is not dense in $m(S)$ [16].

Several other characterizations of ELA semigroups are given in [22], [15], [16].

The class of ELA semigroups is indeed immense and as shown in [15] any left cancellation semigroup can be imbedded in a left cancellation ELA semigroup. Nevertheless no right cancellation ELA semigroup (except the identity semigroup) exists.

Received by the editors November 27, 1967. 
It is the main purpose of this paper to display and prove several functional analytic properties of ELA semigroups some of which turn out to be new characterizations of ELA semigroups (\$2). Theorem 5 (\$2) yields the following beautiful (we think) geometric characterization of ELA semigroups:

A semigroup $S$ is ELA if and only if for any normed space $X$ and any antirepresentation $\left\{T_{s} ; s \in S\right\}$ of $S$ as linear maps from $X$ to $X$ with $\left\|T_{s}\right\| \leqq 1$

$$
\operatorname{dist}(0, O(z))=\operatorname{dist}(0, \text { Co } O(z)) \text { for all } z \in X
$$

where $O(z)=\left\{T_{s} z ; s \in S\right\}$, Co $B$ is the convex hull of $B \subset X$ and

$$
\operatorname{dist}(0, B)=\inf \{\|x\| ; x \in B\} .
$$

This theorem as well as Theorem 6 , on the representations of $S$ as algebra homomorphisms of certain Banach algebras are inspired by a result of I. Glicksberg [30, pp. 99, 104].

Theorem 8 of this section is an analogue for extremely amenable semigroups of the Alaoglu-Birkhoff ergodic theorem, (see Dixmier [6, p. 223]) the main difference being that the convex hull of the orbit of an element is replaced by the orbit itself.

Theorem 7 shows that the class of ELA semigroups coincides with the class of semigroups possessing a kind of "multiplicative invariant extension property" for Banach algebras. It is analogous to a property of amenable semigroups obtained by R. J. Silverman in [24] and turns out to be a characterization of ELA semigroups. The proofs of Theorems 7 and 8 are different from the proofs given in the literature for the amenable case.

Theorem 4 is a generalization of a theorem of Bonsall, Lindenstrauss, and Phelps [1] on the extreme points of the set of linear nonnegative normalized operators between two function algebras and turns out again to be a characterization of ELA semigroups. It does not have an analogue to the left amenable case.

Corollary 3 provides a new purely algebraic characterization of an ELA semigroup $S$ in terms of its antirepresentations $\left\{T_{s} ; s \in S\right\}$ as ring homomorphisms of any (not necessarily commutative) ring $R$ into itself, $S$ being ELA if and only if the linear span of $\left\{\bigcup_{s \in S}\left(I-T_{s}\right) R\right\}$ coincides with $\bigcup_{s \in S} T_{s}^{-1}\{0\}$ (where $I: R \rightarrow R$ is the identity) for any $R$ and each antirepresentation. If $S$ is ELA then the linear span of $\left\{\bigcup_{s \in S}\left(I-T_{s}\right) R\right\}$ will hence be a two-sided ideal of $R$ (and in particular $H=K$ in [16]).

In $\S 3$ of this paper, we study the support functional $q(f)=\sup \phi(f)$ of the set of left invariant means for an ELA semigroup $S$ (where the sup is over all multiplicative left invariant means). We furthermore give some results on left almost convergence. $(f \in m(S)$ is left almost convergent to $c$ iff $\phi(f)=c$ for any left invariant mean $\phi \in m(S)^{*}$.)

In Theorem 6 we give several expressions for $q(f)$ which have partial analogues for the left amenable case. In Theorem 8 we give a characterization of left almost convergence which again has a partial analogue for the left amenable case, the main 
difference being that the convex hull of the orbit of an element is replaced here by the orbit itself. We also give in this section a new characterization of an ELA semigroup $S$ in terms of the functional $p(f)=\lim \sup _{s} f(s)$ on $m(S)$, where the lim sup is with respect to a certain partial order which renders any $S$, such that $a S \cap b S \neq \varnothing$ for all $a, b \in S$, a directed set. This result does not have an analogue for the amenable case.

We give in what follows an example of a semigroup $S$ which is ELA and ERA for which there is a function $f_{0}$ which is left almost convergent to 1 (say) but not right almost convergent. It will follow, partly from Theorem 8, that the norm closure of $\left\{l_{s} f_{0} ; s \in S\right\}$ contains a unique constant function which is 1.1 is also the unique constant function in the pointwise closure of $\left\{r_{s} f_{0} ; s \in S\right\}$ while the norm closure of $\left\{r_{s} f_{0} ; s \in S\right\}$ does not contain any constant. Furthermore the pointwise closure of $\left\{l_{s} f_{0} ; s \in S\right\}$ contains at least two different constant functions.

We end this paper with more examples of ELA semigroups. They should convince the reader that even the class of semigroups which are ELA and ERA and do not possess a zero is immense.

I. Some notations. If $S$ is a semigroup, we denote by LIM (RIM) the set of left (right) invariant means on $m(S)$. LIM (RIM) will also stand as an abbreviation for "left (right) invariant mean."

If a semigroup $S$ is extremely left amenable (ELA) and extremely right amenable (ERA) (i.e. there are multiplicative $\phi, \psi m(S)^{*}$ such that $\phi \in \mathrm{LIM}, \psi \in \mathrm{RIM}$ ) then $S$ is extremely amenable (EA) i.e. there is some multiplicative $\psi_{0} \in$ LIM $\cap$ RIM (for example $\psi_{0}=\phi \odot \psi \in \operatorname{LIM} \cap \operatorname{RIM}$, see Day [4, p. 529], and $\psi_{0}$ is multiplicative).

We shall abbreviate left (right) amenable by LA (RA).

If $S$ is a semigroup and $A \subset S$ we shall sometimes identify $A$ with the set point measures $\left\{1_{a} ; a \in A\right\} \subset \beta(S) \subset m(S)^{*}$ and then $\bar{A}$ the $w^{*}$-closure of $A$ in $\beta(S)$ (or for that matter in $\left.m(S)^{*}\right)$ coincides with $\{\phi \in \beta(S) ; \phi(A)=1\}$ as readily seen and well known. $\left(1_{a}(f)=f(a)\right.$ for all $f \in m(S), a \in S$. $)$

If $X$ is a Banach space with conjugate Banach space $X^{*}$ then the $w$-topology on $X$ (the $w^{*}$-topology on $X^{*}$ ) is the weakest topology on $X$ (on $X^{*}$ ) which renders all $x^{*} \in X^{*}$ (all $\left.x \in X\right)$ continuous linear functionals on $X$ (on $X^{*}$ ).

The relation $\geqq$ renders the set $D$ a directed set [28, p. 65] if: (a) $x \geqq x$ for all $x$ in $D$. (b) If $y \geqq x$ and $z \geqq y$ with $x, y, z \in D$ then $z \geqq x$. (c) For any $x, y \in D$ there is some $z \in D$ such that $z \geqq x$ and $z \geqq y$.

If $S$ is any semigroup such that $s S \cap t S \neq \varnothing$ for all $s, t \in S$ then the relation $t \geqq s$ iff $t \in s S \cup\{s\}$ renders $S$ a directed set ((a) and (c) are clear and if $u \geqq t, t \geqq s$ then $u \in t S \cup\{t\}$ and $t \in s S \cup\{s\}$. So $u \in s S \cup\{s\}$ i.e. $u \geqq s)$.

In particular if $S$ is an amenable semigroup, then $S s \cap S t \neq \varnothing$ and $s S \cap t S \neq \varnothing$ for all $s, t \in S$. Two partial order relations are thus defined in $S$. The right partial order: $t \geqq s$ iff $t \in s S \cup\{s\}$. And the left partial order: $t \geqq s$ iff $t \in S s \cup\{s\}$. Both these partial orders render $S$ a directed set. If then $\left\{y_{s} ; s \in S\right\}$ is a net with values 
in a topological space $Y$ then $(l)-\lim _{s} y_{s}\left[(r)-\lim _{s} y_{s}\right]$ will denote the limit of the net $y_{s}$ with respect to the left [right] partial order of $S$. For any other notation we refer to [15], [16].

II. Some functional analytic properties of ELA semigroups. We will need in what follows the following easy lemma:

LEMma 1. Let $S$ be ELA and $\phi \in m(S)^{*}$ be a multiplicative mean such that $\phi(s S)=1$ for all $s \in S$. If $\left\{1_{s_{\alpha}}\right\} \subset m(S)^{*}$ is any net such that $w^{*}-\lim _{\alpha} 1_{s_{\alpha}}=\phi$, then $\lim _{\alpha}\left\|L_{s} 1_{s_{\alpha}}-1_{s_{\alpha}}\right\|=0$ for all $s \in S$ (i.e. for any $s \in S$ there is some $\alpha_{0}$ with $s s_{\alpha}=s_{\alpha}$ if $\left.\alpha \geqq \alpha_{0}\right)$.

Proof. Let $s \in S$ and choose $t \in S$ such that $s t=t$. Then $1=\phi(t S)=\lim _{\alpha} 1_{t S}\left(s_{\alpha}\right)$. Since $1_{t S} \in m(S)$ is 0,1 , valued there is some $\alpha_{0}$ such that $s_{\alpha} \in t S$ if $\alpha \geqq \alpha_{0}$. Thus $s s_{\alpha}=s_{\alpha}$ if $\alpha \geqq \alpha_{0}$ or $\left\|L_{s} 1_{s_{\alpha}}-1_{s_{\alpha}}\right\|=\left\|1_{s s_{\alpha}}-1_{s_{\alpha}}\right\|=0$ for $\alpha \geqq \alpha_{0}$.

Remarks. 1. Necessarily $\phi$ is a LIM (see Day [4, p. 520(A)]). This lemma yields an easier proof to Theorem 2 on p. 187 of [15].

2. In particular the set of multiplicative LIM's coincides with the set of multiplicative means on $m(S)$ which "live" on right ideals of $S$ (i.e. such that $\phi(s S)=1$ for all $s \in S$ ). This identification of the set of extreme LIM's is due to J. Sorenson and is given in his thesis [25] with different proof.

3. If $S$ is EA and $\phi$ is a multiplicative left and right invariant mean on $m(S)$ and if $\left\{s_{\alpha}\right\} \subset S$ is such that $w^{*}$-lim $1_{s_{\alpha}}=\phi$ then for any $s \in S$ there is some $\alpha_{0}$ such that $s s_{\alpha}=s_{\alpha} s=s_{\alpha}$ if $\alpha \geqq \alpha_{0}$. In particular if $S$ is an EA semigroup of linear bounded maps from the normed space $X$ to $X$ then there is a net $\left\{s_{\alpha}\right\} \subset S$ such that $\lim _{\alpha}\left\|s_{\alpha} s-s_{\alpha}\right\|=\lim _{\alpha}\left\|s s_{\alpha}-s_{\alpha}\right\|=0$ for all $s \in S$ (i.e. $S$ is uniformly ergodic under a net $\left\{s_{\alpha}\right\}$ of elements of $S$. Compare with M. Day [4, p. 536]).

THEOREM 2. (a) Let $G$ be a group with identity $e$ and $\left\{T_{s} ; s \in S\right\}$ be an antirepresentation of the semigroup $S$ as homomorphisms from $G$ to $G$. Let $K_{G}$ be the subgroup of $G$ generated by all elements $\left\{g\left(T_{s} g\right)^{-1} ; g \in G, s \in S\right\}$.

If $S$ is ELA then

$$
K_{G}=\bigcup_{s \in S} T_{s}^{-1}\{e\}
$$

and the set $\left\{g\left(T_{s} g\right)^{-1} ; g \in G, s \in S\right\}$ coincides with the group $K_{G}$.

(b) If $S$ is a semigroup whose antirepresentation $\left\{l_{s} ; s \in S\right\}$ on the additive group $m(S)$ satisfies $(*)$ then $S$ is ELA.

REMARK. If $G$ is a linear space then $K_{G}=\left\{\sum_{1}^{n}\left(g_{i}-T_{s_{i}} g_{i}\right) ; g_{i} \in G, s_{i} \in S, n>0\right\}$ and $K_{G}$ coincides with the $\operatorname{set}\left\{g-T_{s} g ; s \in S, g \in G\right\}$.

Proof. (a) If $T_{s} g=e$ for some $s \in S$ then $g=g\left(T_{s} g\right)^{-1} \in K_{G}$. Conversely if $f \in K_{G}$ then $f=\left[g_{1}\left(T_{s_{1}} g_{1}\right)^{-1}\right]^{k_{1}} \cdots\left[g_{n}\left(T_{s_{n}} g_{n}\right)^{-1}\right]^{k_{n}}$ with $g_{i} \in G, s_{i} \in S$ and integers $k_{i}$. If $s \in S$ satisfies $s_{i} s=s, i \leqq n$, then

$$
T_{s}\left[g_{i}\left(T_{s_{i}} g_{i}\right)^{-1}\right]^{k_{i}}=\left[\left(T_{s} g_{i}\right)\left(T_{s} T_{s_{i}} g_{i}\right)^{-1}\right]^{k_{i}}=\left[\left(T_{s} g_{i}\right)\left(T_{s_{i}} g_{i}\right)^{-1}\right]^{k_{i}}=e
$$


and so $T_{s} f=e$. Consequently $\left({ }^{*}\right)$ holds and even $f=f\left(T_{s} f\right)^{-1}$ i.e.

$$
K_{G}=\left\{g\left(T_{s} g\right)^{-1} ; g \in G, s \in S\right\} .
$$

(b) In this case, $K=K_{m(S)}=\bigcup_{s \in S}\left\{f \in m(S) ; l_{s} f=0\right\}$ and if $f \in K, g \in m(S)$, and $l_{s} f=0$ then $l_{s}(f g)=\left(l_{s} f\right)\left(l_{s} g\right)=0$. Hence $K$ is an ideal of the algebra $m(S)$ and so $K=H=\left\{\sum_{1}^{n} f_{i}\left(g_{i}-l_{s_{i}} g_{i}\right) ; f_{i}, g_{i} \in m(S), s_{i} \in S, 1 \leqq n<\infty\right\}$. But moreover, $H$ is not dense in $m(S)$. Otherwise there would be some $h \in K$ with $\|1-h\|<\frac{1}{2}$. Then $h(s)>\frac{1}{2}$ for all $s \in S$. But then $\left(l_{t} h\right)(s)=h(t s)>\frac{1}{2}$ for all $t, s \in S$. Thus $l_{t} h \neq 0$ for all $t \in S$ which cannot be. By Theorem 2 of [16], $S$ is ELA.

[One cannot replace "antirepresentation" by "representation" in this theorem. Since take $S=\left\{e_{1}, \ldots, e_{n}\right\}$ with $e_{i} e_{j}=e_{i}$ for $1 \leqq i, j \leqq n$ and $n>1$. Then the representation $\left\{r_{s} ; s \in S\right\}$ as (even algebra) homomorphisms from $m(S)$ to $m(S)$ will satisfy $\{0\}=K_{m(S)}=\bigcup_{s \in S} r_{s}^{-1}\{0\}$ as readily checked, but $S$ is not ELA, so (b) does not hold, (here $K_{m(S)}$ is the linear span of $\left\{f-r_{s} f ; f \in m(S), s \in S\right\}$ ).

For (a) consider the ELA semigroup $S^{\prime}=\left\{e_{1}, \ldots, e_{n}\right\}$ with $e_{i} e_{j}=e_{j}$ for $1 \leqq i, j \leqq n$ (where $n>1$ ) and its representation $\left\{r_{s} ; s \in S^{\prime}\right\}$. Let $f \in m\left(S^{\prime}\right)$ be defined by $f\left(e_{j}\right)=j$ for $1 \leqq j \leqq n$. Then $r_{e_{2}} f-r_{e_{1}} f \in K_{m\left(S^{\prime}\right)}$ and $\left(r_{e_{f}} f\right)(s)=f\left(e_{j}\right)$ for all $s \in S^{\prime}$. Thus $r_{a}\left(r_{e_{2}} f-r_{e_{1}} f\right)=1 \in m\left(S^{\prime}\right)$ for all $a \in S^{\prime}$. Thus $K_{m\left(S^{\prime}\right)} \neq \bigcup_{s \in S^{\prime}} r_{s}^{-1}\{0\}$ so (a) does not hold. It is interesting though that the $\operatorname{set}\left\{f-r_{s} f ; f \in m\left(S^{\prime}\right), s \in S^{\prime}\right\}$ coincides with $\bigcup_{s \in S^{\prime}} r_{s}^{-1}\{0\}$ (and in particular both these sets are not linear subspaces of $m\left(S^{\prime}\right)$ as would be the case for antirepresentations). Since $\left[r_{a}\left(f-r_{a} f\right)\right](s)=\left(f-r_{a} f\right)(a)$ $=f(a)-f\left(a^{2}\right)=0$ for any $s \in S^{\prime}$. Conversely if $r_{b} f=0$ then $f=f-r_{b} f \in K_{m\left(S^{\prime}\right)}$, (here $K_{m\left(S^{\prime}\right)}$ stands for the linear span of $\left.\left\{f-r_{s} f ; f \in m\left(S^{\prime}\right), s \in S^{\prime}\right\}\right)$.

Notation. In all that follows $K(H)$ will always stand for the linear span (ideal) generated by $\left\{f-l_{s} f ; f \in m(S), s \in S\right\}$.

Remark. Let $S=S_{n}=\left\{e_{1}, \ldots, e_{n}\right\}$ with $e_{i} e_{j}=e_{i}$ for $i, j \leqq n$. Consider the antirepresentation $R_{s}: m(S)^{*} \rightarrow m(S)^{*}$ of $S$ where $R_{s} \phi(f)=\phi\left(f^{s}\right)$. Since $f=f^{a}$ for all $a \in S, f \in m(S)$ one has that $\phi-R_{a} \phi=0$ for all $\phi \in m(S)^{*}$. Thus $K_{m(S)^{*}}=\{0\}=$ $\bigcup_{s \in S}\left\{\phi \in m(S)^{*} ; R_{s} \phi=0\right\}$ but $S$ is not ELA. This shows that the antirepresentation $\left\{l_{s} ; s \in S\right\}$ over $m(S)$ is distinguished in a sense. It is an antirepresentation of $S$ as algebra homomorphisms from $m(S)$ to $m(S)$.

Corollary 3. Let $A$ be a ring (not necessarily commutatice) and $\left\{T_{s} ; s \in S\right\}$ an antirepresentation of the ELA semigroup $S$ as ring homomorphisms of $A$ into $A$. Then $K_{A}=\left\{\sum_{1}^{n}\left(a_{i}-T_{s_{i}} a_{i}\right) ; a_{i} \in A, s_{i} \in S, n=1,2, \ldots\right\}$ satisfies

$$
K_{A}=\left\{a-T_{s} a ; s \in S, a \in A\right\}=\bigcup_{s \in S} T_{s}^{-1}\{0\} .
$$

Furthermore $K_{A}$ is a two-sided ideal of the ring $A$ which coincides with $H_{A}=$ $\left\{\sum_{1}^{n} b_{i}\left(a_{i}-T_{s_{i}} a_{i}\right) c_{i} ; s_{i} \in S, a_{i}, b_{i}, c_{i} \in A\right.$ (some of $b_{i}$ or $c_{i}$ need not appear), $\left.n=1,2, \ldots\right\}$.

Consequently, if $T$ is an additive map from $A$ to the abelian group $B$ which satisfies $T T_{s} a=T a$ for all $s \in S, a \in A$ then $T\left[b\left(T_{s} a\right) c\right]=T(b a c)$ for all $s \in S, a, b$, $c \in A$, (where $b$ or $c$ need not appear). 
Proof. (**) is just $\left({ }^{*}\right)$ of Theorem 2 applied to the additive group $A$. If $g \in K_{A}$ and $T_{s} g=0$ and $f \in A$ then $T_{s}(f g)=\left(T_{s} f\right)\left(T_{s} g\right)=0=T_{s}(g f)$ thus $K_{A}$ is a two-sided ideal of $A$ and so $K_{A}=H_{A}$. If $T: A \rightarrow B$ is as above then $T\left(H_{A}\right)=T\left(K_{A}\right)=\{0\}$. Hence $T\left[b\left(a-T_{s} a\right) c\right]=0$ for all $s \in S, a, b, c \in A$ (where $b$ or $c$ need not appear).

REMARK. In particular, if $S$ is ELA not only is $\bar{K}=\bar{H}$ as shown in Theorem 6 of [16] (there $A=m(S), H=H_{A}, K=K_{A}$ ) but even $K=H$. Furthermore any linear operator $T$ on $m(S)$ which satisfies $T\left(g_{s}\right)=T(g)$ for all $g \in m(S)$ and $s \in S$ will also satisfy $T\left(f g_{s}\right)=T(f g)$ for all $f, g \in m(S), s \in S$.

EXAMPLES. Let $\mathscr{F}$ be a $\sigma$-field of subsets of $X$ and $S$ be an ELA semigroup of $\mathscr{F}$-measurable maps $s: X \rightarrow X$. (i.e. $s^{-1}(F) \in \mathscr{F}$, if $F \in \mathscr{F}$.) If $\phi$ is an $S$-invariant finitely additive set function on $\mathscr{F}$ (i.e. $\phi\left(s^{-1} P\right)=\phi(P)$ for all $P \in \mathscr{F}, s \in S$ ) then $\phi\left(Q \cap s^{-1} P\right)=\phi(Q \cap P)$ for all $P, Q \in \mathscr{F}$ and $s \in S$. Since let $A$ be the algebra of all simple measurable functions on $X$. Then $\left(T_{s} f\right)(x)=f(s x)$ is an antirepresentation of $S$ as ring homomorphisms of $A$ to $A$. $\phi$ can be viewed as a linear functional on $A$ such that $\phi T_{s} f=\phi f$ for $s \in S, f \in A$. Thus $\phi\left(f\left(T_{s} g\right)\right)=\phi(f g)$. Upon taking $f=1_{P}$, $g=1_{Q}$ one gets the result.

We bring now a generalization of a theorem of Bonsall, Lindenstrauss and Phelps in [1] which will turn out to be a new characterization of ELA semigroups. Let $A, B$ be algebras of bounded real functions on the sets $X$ and $Y$, containing the constants. Let $S$ be a semigroup of maps which acts from $X$ to $X$ i.e. such that $(s t)(x)=s(t x)$ for $x \in X, s, t \in S$. Denote by $K_{S}(A, B)$ the set of linear transformations $T$ from $A$ to $B$ such that $T f \geqq 0$ if $f \geqq 0$ and $T 1=1$ (the constant one functions on $X, Y$ resp.) and such that $T\left(f_{s}\right)=T(f)$ for any $f \in A, s \in S$ where $f_{s}(x)=f(s x)$.

THEOREM 4. If $S$ is ELA then $K_{S}(A, B)$ has extreme points (a fortiori $K_{S}(A, B) \neq \varnothing$ ) and any such extreme point is multiplicative.

REMARK. It is interesting, even though trivial, that this theorem holds only for ELA semigroups. In fact if $S$ is not ELA take $X=S$ with $s(x)=s x, A=m(S)$, and $Y$ to contain just one point, i.e. $B$ to be the field of reals. Then $K_{S}(A, B)$ coincides with the set of LIM's. So, either $K_{S}(A, B)=\varnothing$ or if not then $K_{S}(A, B)$ has an extreme point (by the Krein-Mil'man theorem) which cannot be multiplicative since $S$ is not ELA.

Proof. $\beta(X) \subset m(X)^{*}$ with the $w^{*}$ topology is compact Hausdorff and if $\left(L_{s} \phi\right)(f)$ $=\phi\left(f_{s}\right)$, for $\phi \in \beta X, f \in m(X)$ and $s \in S$, then $L_{s}[\beta(X)] \subset \beta(X)$ and $L_{s}$ are $w^{*}$-continuous and $L_{s} L_{t}=L_{s t}$ for $s, t \in S$. Thus, by Mitchell's fixed point theorem, there is some $\phi \in \beta X$ such that $L_{s} \phi=\phi$ for $s \in S$. The restriction $\phi_{0}$ of $\phi$ to $A$ is a multiplicative mean on $A$ with $\phi\left(f_{s}\right)=\phi f$ for $s \in S, f \in A$. Define $T: A \rightarrow B$ by (Tf) $=\left[\phi_{0}(f)\right] 1$ where $1 \in B$ is the constant one function on $Y$. Then $T \in K_{S}(A, B)$ is multiplicative and thus even an extreme point of the set of all linear $T: A \rightarrow B$ which satisfy $T(1)=1$ and $T f \geqq 0$ if $f \geqq 0$ (see [1, Proposition 15]) and a fortiori of $K_{S}(A, B)$. To show that any extreme $T \in K_{S}(A, B)$ is multiplicative we use the idea in Lemma 1 of Bonsall, Lindenstrauss and Phelps in [1] and the previous corollary. 
Let $T \in K_{S}(A, B)$ be extreme. For $g \in A$ with $0 \leqq g \leqq 1$ define $U_{g}: A \rightarrow B$ by $U_{g}(f)$ $=T(g f)-(T g)(T f)$. Then $U_{g}\left(f_{s}\right)=T\left(g f_{s}\right)-(T g)\left(T f_{s}\right)=U_{g}(f)$ by Corollary 3. Furthermore $U_{g}(1)=0$ thus $\left(T \pm U_{g}\right)(1)=T(1)=1$ and $\left(T \pm U_{g}\right)\left(f_{s}\right)=\left(T \pm U_{g}\right)(f)$ for $f \in m(S)$, $s \in S$. If now $f \in A, f \geqq 0$ then $\left(T+U_{g}\right) f=T(f)+T(f g)-(T f)(T g)=(T f)[1-T g]+$ $T(f g) \geqq 0$ since $0 \leqq g \leqq 1$. Now $\left(T-U_{g}\right)(f)=T[f(1-g)]+(T f)(T g) \geqq 0$ which shows that $T \pm U_{g} \in K_{S}(A, B)$. Since $T$ is extreme $U_{g}=0$ i.e. $(T f)(T g)=T(f g)$ for all $f, g \in A$ with $0 \leqq g \leqq 1$ (thus for any $g \geqq 0$ ). Since $A$ is a lattice one gets that $T$ is multiplicative. (We assume that $A$ is norm closed.)

REMARK. Taking $S$ as the identity semigroup one gets a theorem of Bonsall, Lindenstrauss and Phelps in [1]. Other results of [1] could be generalized in this direction. Some boundedness condition on the functions in $A$ is needed though. Since, if $S$ denotes the semigroup of positive integers with the multiplication $i \vee j=\max \{i, j\}$ and $A$ is the set of all real functions on $S$ and $B=R$ is the field of reals then $K_{S}(A, R)=\varnothing$. For any linear $\phi$ on $A$ such that $\phi(f) \geqq 0$ if $f \geqq 0$ and $\phi(1)=1$ is of the form $\phi(f)=\sum_{i=1}^{k} \alpha_{i} f\left(n_{i}\right)$ where $\alpha_{i} \geqq 0, \sum_{1}^{k} \alpha_{i}=1$. But if $n>n_{1}$ then $l_{n} 1_{\left\{n_{1}\right\}}=0$. Thus $\alpha_{1}=\phi\left(1_{\left\{n_{1}\right\}}\right)=\phi\{0\}=0$. In the same way $\alpha_{i}=0$ for all $i$. Thus $\phi(1)=0$ which cannot be (see also [1, Theorem 8]). Note that this $S$ is ELA.

We give now a geometric characterization of ELA semigroups inspired by a result of Glicksberg [30, p. 99, 104]. The proof is entirely different from [30].

Let $\left\{T_{s} ; s \in S\right\}$ be a (anti)representation of the semigroup $S$ as bounded linear maps on the normed space $X$. Denote by $O(x)=\left\{T_{s} x ; s \in S\right\}$ Co $O(x)$ the convex hull of $O(x)$ and dist $(x, A)=\inf \{\|x-y\| ; y \in A\}$ for all $x \in X$ and $A \subset X . K_{X}$ as above is the linear span of $\left\{x-T_{s} x, x \in X ; s \in S\right\}$. But Co $O(x) \subset x+K_{X}$ (if $y=\sum_{1}^{n} \alpha_{i} T_{s_{i}} x$ with $\sum_{1}^{n} \alpha_{i}=1$. Then $\left.y=x+\sum_{1}^{n} \alpha_{i}\left(T_{s_{i}} x-x\right) \in x+K_{X}\right)$. Thus for any semigroup $S$ :

$$
\operatorname{dist}(0, O(x)) \geqq \operatorname{dist}(0, \text { Co } O(x)) \geqq \operatorname{dist}\left(0, x+K_{X}\right)=\operatorname{dist}\left(x, K_{X}\right) .
$$

THEOREM 5. (I) Let $\left\{T_{s} ; s \in S\right\}$ be an antirepresentation of the semigroup $S$ as linear maps on the normed space $X$ to $X$, with $\left\|T_{s}\right\| \leqq 1$ for all $s \in S$.

(a) If $S$ is $L A$ then dist $(0$, Co $O(x))=\operatorname{dist}\left(x, K_{X}\right)$ for all $x \in X$ (due to Glicksberg [30]).

(b) If $S$ is ELA then even dist $(0, O(x))=\operatorname{dist}(0$, Co $O(x))=\operatorname{dist}\left(x, K_{X}\right)$ for all $x \in X$.

(II) Let $S$ be a semigroup and consider the antirepresentation $\left\{l_{s} ; s \in S\right\}$ over $m(S)$.

(a) If $\left\{l_{s} ; s \in S\right\}$ satisfies dist $(0$, Co $O\{1\})=\operatorname{dist}\left(1, K_{m(S)}\right)$ then $S$ is LA and consequently dist $(0, \mathrm{Co} O(x))=\operatorname{dist}\left(x, K_{m(S)}\right)$ for all $x \in m(S)$ (compare with [18, p. 235]).

(b) If $\left\{l_{s} ; s \in S\right\}$ satisfies dist $(0, O(x))=\operatorname{dist}(0$, Co $O(x))$ for all $x \in m(S)$ then $S$ is $E L A$ and consequently even dist $(0, O(x))=\operatorname{dist}\left(x, K_{m(S)}\right)$ for all $x \in m(S)$.

Proof I (a). We could refer to [30, p. 99 and p. 104]. For the sake of completeness we give a different simple proof based on Day's strong amenability theorem $[4$, 
p. 524] (see also p. 538). For any $\phi \in m(S)^{*}$ which belongs to the linear span of point measures (i.e. $\phi=\sum_{1}^{n} \alpha_{i} 1_{s_{i}}$ for some $\left\{s_{1}, \ldots, s_{n}\right\} \subset S$ ) define $T_{\phi} x=\sum_{1}^{n} \alpha_{i} T_{s_{1}} x$, for all $x \in X$. Then $\left\|T_{\phi} x\right\| \leqq \sum_{1}^{n}\left|\alpha_{i}\right|\|x\|=\|\phi\|\|x\|$. Now $\left\|T_{\phi}\left(x-T_{a} x\right)\right\|=\left\|\left(\sum \alpha_{i} T_{s_{i}}-\sum \alpha_{i} T_{a s_{i}}\right) x\right\|$ $=\left\|T_{\phi-L_{a} \phi} x\right\| \leqq\left\|\phi-L_{a} \phi\right\|\|x\|$, for all $x \in X$.

Let now $\left\{\phi_{a}\right\} \subset m(S)^{*}$ be a net of finite means (i.e. $\phi_{\alpha} \in \operatorname{Co}\left\{1_{s} ; s \in S\right\}$ ) strongly converging to left invariance (i.e. such that $\left\|L_{a} \phi_{\alpha}-\phi_{\alpha}\right\| \rightarrow 0$ for all $a \in S[4$, p. 524]. Then $\left\|T_{\phi_{\alpha}}\left(x-T_{a} x\right)\right\| \rightarrow 0$ for all $x \in X$ and $a \in S$. Thus $\left\|T_{\phi_{\alpha}} y\right\| \rightarrow 0$ for $y \in K_{X}$. Notice also that $T_{\psi} x \in \mathrm{Co} O(x)$ for any finite mean $\psi$.

Let now $z \in X, \varepsilon>0$ and $h \in K_{X}$ satisfy $\|z+h\|<\operatorname{dist}\left(z, K_{X}\right)+\varepsilon$. Choose $\beta$ such that $\left\|T_{\phi_{\beta}} h\right\|<\varepsilon$. Then

$$
\left\|T_{\phi_{\beta}} z\right\| \leqq\left\|T_{\phi_{\beta}}(z+h)\right\|+\left\|T_{\phi_{\beta}} h\right\|<\operatorname{dist}\left(z, K_{X}\right)+2 \varepsilon .
$$

Hence dist $(0$, Co $O(z)) \leqq \operatorname{dist}\left(z, K_{X}\right)$ and $\mathrm{I}(\mathrm{a})$ holds.

I(b). If $y \in K_{X}$ then $T_{s} y=0$ for some $s \in S$ by Theorem 2(a). Thus $\|x+y\|$ $\geqq\left\|T_{s}(x+y)\right\|=\left\|T_{s} x\right\|$ so dist $\left(x, K_{X}\right) \geqq \operatorname{dist}(0, O(x)) \geqq \operatorname{dist}\left(x, K_{X}\right)$.

II(a). If $S$ is not LA then $\inf \left\{\|1-h\| ; h \in K_{m(S)}\right\}<1[18$, p. 235] while

$$
\operatorname{dist}(0, \operatorname{Co} O\{1\})=1 \text {. }
$$

Invoke now I(a).

II(b). We show at first that $A \cap B \neq \varnothing$ for any two nonvoid right ideals $A, B$ of $S$. If not let $A, B$ be two nonvoid right ideals with $A \cap B=\varnothing$. Let $a \in A, b \in B$ and $f=1_{A}-1_{B}$. Then $f_{a}=1, f_{b}=-1$ so $0=\frac{1}{2}\left(f_{a}+f_{b}\right)$. Thus $0=\operatorname{dist}(0, \operatorname{Co} O(f))$ $=\operatorname{dist}(0, O(f))$. So there is some $c \in S$ such that $\left\|l_{c} f\right\|<\frac{1}{2}$. Since $f$ takes only the values \pm 1 and 0 this shows that $l_{c} f=0$ i.e. that $1_{A}(c s)=1_{B}(c s)$ for all $s \in S$. If $t \in S$ satisfies $c t \in A$ (or $c t \in B$ ) then $1=1_{A}(c t)=1_{B}(c t)$ so $c t \in B(c t \in A)$ which cannot be since $A \cap B=\varnothing$ therefore there is a nonvoid right ideal $C$ (namely $c S$ ) such that $C \cap(A \cup B)=\varnothing$.

Let now $A_{0}, B_{0}$ be two fixed disjoint nonvoid right ideals. By an easy application of Zorn's lemma there is a family of pairwise disjoint right ideals $\mathscr{R}$ to which $A_{0}$ and $B_{0}$ belong which is maximal with respect to the property of pairwise disjointness (i.e. any other right ideal of $S$ intersects some element of $\mathscr{R}$ ).

Let $A=A_{0}$ and $B$ be the union of all other right ideals of $\mathscr{R}$. Then $B_{0} \subset B$ and $A, B$ are nonvoid right ideals with $A \cap B=\varnothing$. Let $C \neq \varnothing$ be a right ideal with $C \cap(A \cup B)=\varnothing$. Then $\mathscr{R}_{1}=\mathscr{R} \cup\{C\}$ properly includes $\mathscr{R}$ and all elements of $\mathscr{R}_{1}$ are pairwise disjoint, which contradicts the maximality of $\mathscr{R}$. This contradiction shows that any two (and hence any finite collection of) right ideals have nonvoid intersection.

If now $f \in m(S), a \in S$ and $\varepsilon>0$ then

$$
\left\|\frac{1}{n}\left(\sum_{1}^{n} l_{a^{i}}\right)\left(f-l_{a} f\right)\right\|=\frac{1}{n}\left\|l_{a} f-l_{a^{n+1}} f\right\|<\frac{2}{n}\|f\|<\varepsilon
$$

if $n$ is big. Thus dist $\left(0, \operatorname{Co} O\left(f-f_{a}\right)\right)=0=\operatorname{dist}\left(0, O\left(f-f_{a}\right)\right)$. So, $\left\|l_{c s}\left(f-f_{a}\right)\right\|$ $\left\|l_{s} l_{c}\left(f-f_{a}\right)\right\| \leqq\left\|l_{c}\left(f-f_{a}\right)\right\|<\varepsilon$ for some $c \in S$ and all $s \in S$. Let now $h=\sum_{1}^{n} g_{i}\left(f_{i}-l_{a_{i}} f_{i}\right)$ 
for some $f_{i}, g_{i} \in m(S), a_{i} \in S$. Choose $c_{i} \in S$ with $\left\|l_{c_{i}}\left(f_{i}-l_{a_{i}} f_{i}\right)\right\|<\varepsilon / n k$ where $k=\max \left(\left\|g_{1}\right\|, \ldots,\left\|g_{n}\right\|\right)$. Choose $s_{1}, \ldots, s_{n} \in S$ such that $c_{1} s_{1}=c_{2} s_{2}=\cdots=c_{n} s_{n}=c$. Then

$$
\left\|l_{c} h\right\|<\left(\max _{1 \leqq i \leqq n}\left\|g_{i}\right\|\right) \sum_{1}^{n}\left\|l_{c}\left(f_{i}-l_{a_{i}} f_{i}\right)\right\|<\varepsilon .
$$

So $\|1-h\| \geqq\left\|l_{c}(1-h)\right\|=\left\|1-l_{c} h\right\|>1-\varepsilon$ i.e. $\|1-h\| \geqq 1$ for all $h \in H=H_{m(S)}$. Hence $\inf \{\|1-h\| ; h \in H\}=1$. Theorem 2 of [16] implies now that $S$ is ELA.

REMARKS. 1. If the hypothesis of II(b) would be replaced by the stronger assumption that dist $(0, O(x))=\operatorname{dist}\left(x, K_{m(S)}\right)$ for all $x \in m(S)$ then the following easier proof can be provided: Since $1=\operatorname{dist}(0, O\{1\})=\inf \left\{\|1-h\| ; h \in K_{m(S)}\right\}, K_{m(S)}$ is not dense in $m(S)$. If now $f \in K_{m(S)}$ and $g \in m(S)$ is arbitrary then

$$
\operatorname{dist}\left(f g, K_{m(S)}\right)=\inf _{s}\left\|l_{s} f l_{s} g\right\| \leqq\|g\| \inf \left\|l_{s} f\right\|=\|g\| \operatorname{dist}\left(f, K_{m(S)}\right)=0 .
$$

Thus $f g \in \bar{K}_{m(S)}$ and $\bar{K}_{m(S)}$ is an ideal which is not dense in $m(S)$ and contains $H_{m(S)}$. By Theorem B in [16] $S$ is ELA.

2. One can now state the following beautiful geometric characterization of ELA semigroups:

THEOREM. A semigroup $S$ is ELA if and only if it enjoys the following geometric property.

(G) For any normed space $X$ and any antirepresentation $\left\{T_{s} ; s \in S\right\}$ of $S$ as linear maps from $X$ to $X$ with $\left\|T_{s}\right\| \leqq 1$, for all $s \in S$, dist $(0, O(x))=\operatorname{dist}(0$, Co $O(x))$ holds for all $x \in X$. For $S$ to be ELA, it suffices that $(\mathrm{G})$ holds for the antirepresentation $\left\{T_{s}=l_{s} ; s \in S\right\}$ over $m(S)$.

We find it striking that the geometric property $(G)$ is equivalent to the algebraic property that each $s, t \in S$ admit a common right zero, or to the topological property that $S$ has the common fixed point property on compacta.

3. Let $G$ be any amenable group with identity $e$ and $G \neq\{e\}$. We show that any left translation invariant subalgebra $A \subset m(G)$ which contains a nonconstant function $f$ also contains some function $h$ with dist $(0, O(h)) \neq \operatorname{dist}(0, \mathrm{Co} O(h))$. Since if $f \in A$ satisfies $f(a) \neq f(b)$ where $a, b \in G$ and $c=a b^{-1}$ then $h=f-f_{c}$ will satisfy $h(b) \neq 0$ so $0 \neq\|h\|=\left\|l_{s} h\right\|$ for all $s \in G$. Thus $0 \neq \operatorname{dist}(0, O(h))$. But $h \in K_{m(s)}$ so $0=\operatorname{dist}\left(h, K_{m(S)}\right)=\operatorname{dist}(0, \operatorname{Co} O(h))$ by $\mathrm{I}(\mathrm{a})$.

Consider the group $Z$ of additive integers. Let $x \in m(Z)$ be defined by $x(n)$ $=(-1)^{n}$. Then $\left\|l_{n} x\right\|=\|x\|=1$ for all $n \in Z$ so dist $(0, O(x))=1$. But $\frac{1}{2}\left(x+l_{1} x\right)=0$ so dist $(0, \operatorname{Co} O(x))=0$.

4. It follows under the assumptions of I(a) that for any $x \in X$ with dist $(0$, Co $O(x))$ $=c>0$, there is some $x^{*} \in X^{*}$ with $\left\|x^{*}\right\|=1, x^{*}\left(K_{X}\right)=0$ and $\left|x^{*}(x)\right|=c=\operatorname{dist}\left(x, K_{X}\right)$. (Invoke the Hahn-Banach theorem.) This is again a result of I. Glicksberg [30]. In partial analogy to it one has

THEOREM 6. Let $S$ be ELA and $\left\{T_{s} ; s \in S\right\}$ be an antirepresentation of $S$ as linear multiplicative maps from the complex commutative Banach algebra $X$ to $X$. Assume 
also that $X$ has identity and that $\|x\|=\sup _{x^{*} \in \cdot M}\left|x^{*}(x)\right|$ for all $x \in X$ where $\mathscr{M}$ is the set of all multiplicative $x^{*} \in X^{*}$.

Then for all $x \in X$

$$
\operatorname{dist}(0, O(x))=\max _{x^{*} \in \mathscr{H}^{\prime} s}\left|x^{*}(x)\right|
$$

where $\mathscr{M}_{S}=\left\{x^{*} \in \mathscr{M} ; T_{s}^{*} x^{*}=x^{*}\right.$ for all $\left.s \in S\right\}$.

This property characterizes ELA semigroups.

Proof. Our conditions imply that $\left\|T_{s}\right\| \leqq 1$ for all $s \in S$, and that $\mathscr{M} \subset X^{*}$ and hence $\mathscr{M}_{S}$ are compact hausdorff in the $w^{*}$ topology.

Let $\psi \in m_{c}(S)^{*}$ be multiplicative on $m_{c}(S)$ (all complex bounded functions on $S$ ) and $x^{*} \in \mathscr{M}$. Then $\psi \odot x^{*} \in X^{*}$ defined by $\left(\psi \odot x^{*}\right)(x)=\psi\left[x^{*}\left(T_{s} x\right)\right]$ belongs to $\mathscr{M}_{s}$, if $\psi$ is left invariant (i.e. if $L_{s} \psi=\psi$ for all $s \in S$ ). This is readily checked. Then

$$
\begin{aligned}
\operatorname{dist}(0, O(x)) & \left.=\inf _{s} \sup _{x^{*} \in \mathscr{M}}\left|x^{*}\left(T_{s} x\right)\right| \leqq \inf \sup _{s} \sup _{t} \mid x^{*} \in T_{s t} x\right) \mid \\
& =\sup _{x^{*} \in \mathscr{M}}\left[\inf _{s} \sup _{t}|F(s t)|\right]
\end{aligned}
$$

where $F(u)=x^{*}\left(T_{u} x\right)$ for $u \in S$.

By Theorem 6 of the next section $\inf _{s} \sup _{t}|F(s t)|=\sup _{\phi} \phi(|F|)$ where $\phi$ ranges over all multiplicative LIM's $\phi$ on $m(S)$ (see Theorem A. 5 in the introduction of [16]). But if $\phi$ is a multiplicative LIM on $m(S)$ then $\phi_{c} \in m_{c}(S)^{*}$ defined by $\phi_{c}(f+i g)=\phi(f)+i \phi(g)$ for $f, g \in m(S)$ is multiplicative on $m_{c}(S), \phi_{c}\left(l_{s} G\right)=\phi_{c}(G)$ for all $s \in S, G \in m_{c}(S)$ and $\phi_{c}(G)=\phi(G)$ if $G \in m(S)$. Since any multiplicative $\psi \in m_{c}(S)$ satisfies $|\psi(G)|=\psi(|G|)$ one has

$$
\begin{aligned}
\operatorname{dist}(0, O(x)) & =\sup _{x^{*} \in \mathscr{H}} \sup _{\phi} \phi(|F|)=\sup _{x^{*} \in \mathscr{H}} \sup _{\phi}\left|\phi_{c}(F)\right| \\
& =\sup _{x^{*} \in \mathscr{M}} \sup _{\phi}\left|\left(\phi_{c} \odot x^{*}\right)(x)\right| \leqq \sup _{y^{*} \in \mathscr{H}_{s}}\left|y^{*}(x)\right| \\
& =\max _{y^{*} \in \mathscr{H}_{s}}\left|y^{*}(x)\right| \leqq \operatorname{dist}(0, O(x))
\end{aligned}
$$

(where $\sup _{\phi}$ denotes sup over all multiplicative LIM's $\phi$ on $m(S)$ ), since $y^{*}(x)$ $=y^{*}(y)$ for any $y^{*} \in \mathscr{M}_{S}$ and $y \in O(x)$. Hence for any $x \in X$ there is some $x^{*} \in \mathscr{M}_{S}$ with $\left|x^{*}(x)\right|=\operatorname{dist}(0, O(x))$. (Notice that $0 \in \mathscr{M}_{S}$ according to our definition of $\mathscr{M}$ and $\mathscr{M}_{S}$.)

Considering the representation $\left\{l_{s} ; s \in S\right\}$ over $m_{c}(S)$ and assuming the theorem to hold for $X=m_{c}(S)$ one has

$$
1=\operatorname{dist}(0, O\{1\})=\max _{\phi \in \mathscr{I}_{s}}|\phi(1)|=\left|\phi_{0}(1)\right| \text { for some } 0 \neq \phi_{0} \in \mathscr{M}_{S}
$$

where $\mathscr{M}_{S}$ is the set of multiplicative left invariant $\phi \in m_{c}(S)^{*} . \phi_{0}$ restricted to $m(S)$ will be a multiplicative LIM as readily seen.

RemarKs. 1. Upon taking $S$ as the identity semigroup in Theorem 6 one has $O(x)=\{x\}$ so $\|x\|=\operatorname{dist}(0, O(x))=\max _{x^{*} \in \mathscr{U}}\left|x^{*}(x)\right|\left(\mathscr{M}=\mathscr{M}_{S}\right.$ in this case $)$. Hence, the assumption $\|x\|=\sup _{x^{*} \in . \|}\left|x^{*}(x)\right|$ for all $x \in X$, cannot be relaxed. 
2. Under the assumptions of Theorem 6, consider the linear span $K_{X}$ of $\left\{x-T_{s} x ; s \in S, x \in X\right\}$. Then $K_{X}=H_{X}$ is an ideal of the commutative Banach algebra $X$ (Corollary 3 ). The space of maximal ideals of the quotient Banach algebra $A=X / \bar{H}_{X}$ coincides with the set of multiplicative $\phi \in X^{*}$ which satisfy $\phi\left(H_{X}\right)=0$ $\left[20\right.$, p. 63] which is just $\mathscr{M}_{S}$. Denoting by $\tilde{x}=x+\bar{H}_{X}$ one has for the norm in $A$,

$$
\|\tilde{x}\|=\inf \left\{\|x+h\|, h \in H_{X}\right\}=\operatorname{dist}(0, O(x))=\operatorname{dist}(0, \text { Co } O(x))=\max _{\phi \in M_{s}}|\phi(x)| \text {. }
$$

Thus the Gelfand homomorphism of $A$ onto its image $\hat{A}$ is an isometry and $\hat{A}=C_{c}\left(\mathscr{M}_{S}\right)=$ all complex continuous functions on $\mathscr{M}_{S} \subset X^{*}$ with the $w^{*}$ topology.

In particular let $X=m_{c}(S), T_{s}=l_{s}: m_{c}(S) \rightarrow m_{c}(S)$. Then

$$
H_{X}=H_{c}=\left\{f-l_{s} f ; s \in S, f \in m_{c}(S)\right\}
$$

(Corollary 3). Hence the image of $m_{c}(S) / \bar{H}_{c}$ under the Gelfand homomorphism coincides with $C_{c}\left(\mathscr{M}_{S}\right)$ where $\mathscr{M}_{S}$ can be identified with the set LIM $\cup\{0\} \subset \tilde{m}(S)^{*}$ in the $w^{*}$ topology (via the extension to $m_{c}(S)$ given by $\phi(f+i g)=\phi(f)+i \phi(g)$ ). Furthermore for any $f \in m_{c}(S)$ one has

$$
\inf _{s} \sup _{t}|f(t s)|=\inf _{h \in H_{c}} \sup _{t}|f(t)+h(t)|=\max |\psi(f)|=\inf _{\mathscr{A}} \sup _{t}\left|\sum_{1}^{n} \alpha_{i} f\left(a_{i} t\right)\right|
$$

where the max is over all multiplicative left invariant $\psi \in m_{c}(S)^{*}$ and

$$
\mathscr{A}=\left\{\left(\alpha_{1}, \ldots, \alpha_{n}, a_{1}, \ldots, a_{n}\right), \alpha_{i} \geqq 0, \sum \alpha_{i}=1, a_{i} \in S, n=1,2, \ldots\right\} .
$$

Another characterization of ELA semigroups is given in terms of a "multiplicative invariant extension property" below. It is partly analogous to a property of amenable semigroups given by R. J. Silverman [24, p. 75]:

TheOREM 7. Let $\left\{T_{s} ; s \in S\right\}$ be an antirepresentation of the ELA semigroup $S$ as continuous algebra homomorphisms from the Banach algebra $B$ into $B$. Let $A \subset B$ be a subalgebra such that $T_{s}(A) \subset A$ for all $s \in S$. Let $\phi \in A^{*}$ be multiplicative and satisfy $\phi\left(T_{s} x\right)=\phi(x)$ for all $x \in A$ and $s \in S$. If there is some multiplicative $\psi \in B^{*}$ which extends $\phi$ then there is a multiplicative extension $\psi_{0} \in B^{*}$ of $\phi$ for which $\psi_{0}\left(T_{s} y\right)=\psi_{0}(y)$ for all $y \in B$ and $s \in S$. Conversely, any semigroup which has this "multiplicative invariant extension property" in necessarily ELA.

Proof. Let $\mathscr{K}=\left\{\psi \in B^{*} ; \psi\right.$ is a multiplicative extension of $\left.\phi\right\}$. Then $\mathscr{K}$ is a $w^{*}$-closed subset of the unit ball of $B^{*}$ and is hence $w^{*}$-compact. Each $T_{s}^{*}: B^{*} \rightarrow B^{*}$ is $w^{*}$-continuous and $T_{s}^{*} T_{t}^{*}=T_{s t}^{*}$ so $\left\{T_{s}^{*} ; s \in S\right\}$, as a homomorphic image of $S$, is ELA. Furthermore $T_{s}^{*}\left(\mathscr{K}^{r}\right) \subset \mathscr{K}$ for all $s \in S$. Since if $\psi \in \mathscr{K}, x \in A, y, z \in B$ then $\left(T_{s}^{*} \psi\right)(x)=\psi\left(T_{s} x\right)=\phi\left(T_{s} x\right)=\phi(x)$ and $\left(T_{s}^{*} \psi\right)(y z)=\psi\left[\left(T_{s} y\right)\left(T_{s} z\right)\right]=\left(T_{s}^{*} \psi\right)(y)\left(T_{s}^{*} \psi\right)(z)$. Thus $T_{s}^{*} \psi \in \mathscr{K}$. By Mitchell's fixed point theorem there is some $\psi_{0} \in \mathscr{K}$ such that $T_{s}^{*} \psi_{0}=\psi_{0}$ for all $s \in S . \psi_{0}$ is the required extension.

Let now $S$ be a semigroup, $B=m(S)$, and $A$ be the algebra of all constant functions in $B$. Let $T_{s}=l_{s}: B \rightarrow B$ for $s \in S$ and define $\phi$ on $A$ by $\phi(c 1)=c$. If $a \in S$ 
is fixed, define $\psi \in m(S)^{*}$ by $\psi(f)=f(a)$ for $f \in m(S)$. Then $\psi$ is a multiplicative extension of $\phi$. If $S$ has the multiplicative invariant extension property then there is a multiplicative $\psi_{0} \in m(S)^{*}$ which extends $\phi$ and such that $\psi_{0}\left(f_{s}\right)=\psi_{0}(f)$ for $s \in S, f \in m(S) . \psi_{0} \neq 0$ since $\psi_{0}(1)=\phi(1)=1 . \psi_{0}$ is necessarily a mean and so $S$ is ELA.

The next theorem is the analogue to EA semigroups of the Birkhoff-Alaoglu ergodic theorem (see Dixmier [6, pp. 223-224]). The proof given is short and straightforward. We remind the reader that, if $y_{s}$ is a net on $S$ with values in the Banach space $Y$ the norm $(l)-\lim _{s} y_{s}\left[\operatorname{norm}(r)-\lim _{s} y_{s}\right]$ denotes the limit, in the norm topology, of the net $y_{s}$, with respect to the left [right] partial order in $S$.

TheOREM 8. Let $X$ be a (real or complex) Banach space and $\left\{T_{s} ; s \in S\right\}$ be a [anti]representation of the EA semigroup $S$ as linear bounded maps from $X$ to $X$, such that $\left\{T_{s} x ; s \in S\right\}$ is w-conditionally compact for all $x \in X$. Then norm $(l)-\lim _{s} T_{s} x$ $=P . x\left[\operatorname{norm}(r)-\lim _{s} T_{s} x=P x\right]$ exists for all $x \in X, P$ is a linear bounded projection onto $F=\left\{x \in X ; T_{s} x=x\right.$ for all $\left.s \in S\right\}$ and $P^{-1}\{0\}=\bar{K}_{X}=$ norm closure of

$$
\left\{x-T_{s} x ; x \in X, s \in S\right\} \text {. }
$$

(Thus $X=F \oplus \bar{K}_{X}$.) Furthermore, for any $x \in X$, the closed convex hull of $\left\{T_{s} x ; s \in S\right\}$ (denoted by $C(x)$ ) intersects $F$ in the unique point $P x$.

Proof. Assume that $\left\{T_{s} ; s \in S\right\}$ is a representation and $X$ is complex. Then, since each $T_{s}: X \rightarrow X$ is $w-w$ continuous [7, p. 422] $T_{a}$ [w-closure of $\left\{T_{s} x ; s \in S\right\}$ ] $\subset w$-closure of $\left\{T_{s} x ; s \in S\right\}$, which is w-compact. By Mitchell's fixed point theorem there is some $x_{0} \in w$-closure of $\left\{T_{s} x ; s \in S\right\} \subset C(x)$ such that $T_{s} x_{0}=x_{0}$ for all $s \in S$. Hence $C(x) \cap F \neq \varnothing$ for all $x \in X$. Let now $y \in C(x) \cap F$ be arbitrary, $\beta=\sup _{s}\left\|T_{s}\right\|$ $<\infty$ [7, p. 53] and $\varepsilon>0$. Let $\sum_{1}^{n} \beta_{i}\left(T_{s_{i}} x\right)$ be a convex combination such that $\left\|y-\sum_{1}^{n} \beta_{i} T_{s_{i}} x\right\|<\varepsilon / \beta$. Let $a \in S$ satisfy $a s_{i}=a, 1 \leqq i \leqq n$. Then for any $s \in S a \cup\{a\}$, $T_{s} T_{s_{i}}=T_{s}$ and so:

$$
\left\|T_{s} x-y\right\|=\left\|T_{s} x-T_{s} y\right\|=\left\|T_{s}\left(\sum \beta_{i} T_{s_{i}} x-y\right)\right\|<\beta \varepsilon / \beta=\varepsilon .
$$

By definition then norm (l)- $\lim _{s} T_{s} x$ exists and equals $y$ where $y \in C(x) \cap F$ is arbitrary. Hence $C(x) \cap F$ contains a unique element, denoted by $P . x$, and $P x=$ norm $(l)-\lim _{s} T_{s} x$ for $x \in X$. Thus $P$ is a linear map $\|P\| \leqq \sup _{s}\left\|T_{s}\right\|$ and $P x=x$ if $x \in F$. But $P\left(T_{a} x\right) \in C\left(T_{a} x\right) \cap F \subset C(x) \cap F=\{P x\}$ and $T_{a}(P x) \subset T_{a}[C(x)]$ $\cap T_{a}(F) \subset C(x) \cap F=\{P x\}$. So $T_{a} P=P T_{a}=P$ for all $a \in S$. Hence $P X=F$ (so $\left.P^{2}=P\right)$ and $F=\{x \in X ; P x=x\}$. Furthermore $P\left(x-T_{s} x\right)=0$ so $\bar{K}_{X} \subset P^{-1}\{0\}$. If now $x$ is such that $P x=0$ then $x=x-P x$ is in the norm closure $\bar{K}_{X}$ of the set $K_{X}=\left\{x-T_{s} x ; s \in S\right\}$. Thus $P^{-1}\{0\}=\bar{K}_{X}$.

If now $y \in X$ then $y=P y+(y-P y)$. Thus $X=P X \oplus P^{-1}\{0\}=F \oplus \bar{K}_{X}$ since $P(X) \cap P^{-1}\{0\}=\{0\}$.

RemarKS. 1. Let $S=\left\{e_{1}, \ldots, e_{n}\right\}, n>1$, with $e_{i} e_{j}=e_{j}$ for $i, j \leqq n$. Let $f_{0} \in m(S)$ be defined by $f_{0}\left(e_{j}\right)=j, 1 \leqq j \leqq n$. Then $r_{e_{j}} f_{0}(s)=j$ for $s \in S$. Thus for the representa- 
tion $\left\{r_{s} ; s \in S\right\}$ over $X=m(S)$ one has that $\left\{r_{s} f_{0} ; s \in S\right\} \cap F$ coincides with the $n>1$ constant functions $\{1,2 \cdot 1, \ldots, n \cdot 1\}$. Thus $C\left(f_{0}\right) \cap F$ contains more than one point. This comes about, since $S$ is ELA but not ERA (not even RA). We see that the assumptions on $S$ in the above theorem cannot be entirely dropped. They can though be relaxed to: $S$ is ERA and LA. We conjecture though, that any ERA and LA semigroup is necessarily ELA. In fact if $S$ is ERA and LA but not ELA then the semigroup $S /(r)$ (see [14, p. 371]) would be a right cancellation ERA and LA semigroup which contains at least two elements. It would thus be a counterexample to a conjecture of Sorenson (and also to one made by us in Bull. Amer. Math. Soc. 72 (1966), p. 1031 which we believe to hold true).

THeOREM 9. Let $X$ be a Banach algebra, $\left\{T_{s} ; s \in S\right\}$ a representation of the EA semigroup $S$ as algebra homomorphisms from $X$ to $X$. Assume that $\left\{T_{s} x ; s \in S\right\}$ is w-conditionally compact for any $x \in X$. Then $P x=\operatorname{norm}(l)-\lim _{s} T_{s} x$ exists for all $x \in X, P$ is a bounded multiplicative projection onto the closed subalgebra

$$
F=\left\{x ; T_{s} x=x, \text { for all } s \in S\right\},
$$

$P^{-1}\{0\}=\bar{K}_{X}=$ norm closure of $\left\{x-T_{s} x ; x \in X, s \in S\right\}$ is a closed two-sided ideal and $X=F \oplus \bar{K}_{X}$.

Proof. By Corollary 3, $K_{X}$ is a two-sided ideal and since $T_{s}$ are bounded and multiplicative $F$ is a closed subalgebra. Furthermore, if $x, y \in X$ then $P(x y)=$ norm $(l)-\lim _{s} T_{s}(x y)=\operatorname{norm}(l)-\lim _{s}\left(T_{s} x\right)\left(T_{s} y\right)=(P x)(P y)$. (Notice that $X$ need not be commutative.)

An extension of the concept of a multiplicative invariant mean which is analogous to the result of Dixmier [6, p. 219] for the left amenable case is given in the following theorem.

TheOREM 10. Let $S$ be a family of maps from $X$ to $X$. Let $A \subset m(X)$ be an $S$ invariant subalgebra with $1 \in A$, which admits an S-invariant multiplicative mean. Let $B$ be a semisimple complex commutative Banach algebra. Let $\mathscr{A}_{A}^{B}(X)=\mathscr{A}$ be a $S$-invariant algebra of functions $f: X \rightarrow B$ for which:

(a) $\psi[f(x)] \in A_{c}=A+i A$ for all $\psi \in B^{*}$ and $f \in \mathscr{A}$,

(b) The w-closure of $\{f(x) ; x \in X\} \subset B$ is w-compact. Then there exists a linear operator $T: \mathscr{A}_{A}^{B}(X) \rightarrow B$ with satisfies for any $f, g \in \mathscr{A}, s \in S: 1$. Tf $\in$ w-closure $\{f(x) ; x \in X\}$. 2. $T(f g)=(T f)(T g)$ and 3. $T f_{s}=T f\left(\right.$ where $\left.f_{s}(x)=f(s(x)), x \in X, s \in S\right)$.

Proof. Let $\mu_{1}$ be a multiplicative $S$-invariant mean on $A \subset m(X)$ and define $\mu$ on $A_{c}=A+i A \subset m_{c}(X)$ by: $\mu(f+i g)=\mu_{1}(f)+i \mu_{1}(g)$ for $f, g \in A$. Then $\mu \in A_{c}^{*}$ is a multiplicative $S$-invariant extension of $\mu_{1}$. Let now $\left\{x_{\alpha}\right\} \subset X$ be a net such that $\mu(F)=\lim _{\alpha} F\left(x_{\alpha}\right)$ for all $F \in A_{c}$. (If $A_{c}$ would separate the points of $X$ this would follow from Corollary 19, p. 276 and Lemma 25 on p. 278 in [7]. If $A_{c}$ does not separate points then $A_{c}$ can be viewed as a point separating algebra on the set of equivalence classes of $x$ by the relation: $x \sim y$ iff $F(x)=F(y)$ for all $F \in A_{c}$. One 
readily gets that a net $\left\{x_{\alpha}\right\} \subset X$ as above again exists.) Hence $\mu[\psi(f(x))]=\lim _{\alpha} \psi\left(f\left(x_{\alpha}\right)\right)$ for any $\psi \in B^{*}$ and $f \in \mathscr{A}$.

Now for fixed $f \in \mathscr{A}$ there is a subnet $x_{\alpha_{\beta}}$ such that $w$-lim $f\left(x_{\alpha_{\beta}}\right)=x_{f}$, for some $x_{f} \in B$. Hence

$$
\lim _{\beta} \psi\left(f\left(x_{\alpha_{\beta}}\right)\right)=\psi\left(x_{f}\right)=\lim _{\alpha} \psi\left(f\left(x_{\alpha}\right)\right) \text { for } \psi \in B^{*},
$$

i.e. $w$ - $\lim f\left(x_{\alpha}\right)=x_{f}$ exists for all $f \in \mathscr{A}$. Define now $T f=x_{f}$. Then

$$
\psi(T f)=\lim _{\alpha} \psi\left(f\left(x_{\alpha}\right)\right)=\mu[\psi(f(x))]
$$

and

$$
\psi\left(T f_{s}\right)=\mu\left[\psi\left(f_{s}(x)\right)\right]=\mu[\psi(f(x))]=\psi(T f) \text { for all } \psi \in B^{*}, s \in S, f \in \mathscr{A} .
$$

Furthermore $T$ is linear (even $\|T f\| \leqq \sup _{x}\|f(x)\|$ ) hence 1 and 3 hold. But $T$ is even multiplicative. Let $\phi \in B^{*}$ be multiplicative, then

$$
\phi(T(f g))=\mu[\phi(f(x) g(x))]=\mu[\phi(f(x))] \mu[\phi(g(x))]=\phi(T f) \phi(T g)=\phi(T f T g)
$$

for $f, g \in \mathscr{A}$. But $B$ is commutative and semisimple and so the multiplicative $\phi \in B^{*}$ separate the points of $B$. Hence $T(f g)=T f T g$ and 3 holds.

REMARKS. 1. If $S$ is ELA one can take $A=m(X)$ hence condition (a) can be dropped.

2. If $S$ is generated by one element $s$ and $X=S$ and if $\lim _{n \rightarrow \infty} \psi\left[f\left(s^{n}\right)\right]$ exists for all $f \in \mathscr{A}$ and $\psi \in B^{*}$ then one can take as $A=c$ the space of real convergent sequences and condition (a) of the theorem holds since $c \subset m$ is ELA.

3. Take $X=S, A=m(S)$, and $B=R$ the field of reals. Then $\mathscr{A}=m(S)$. If an operator $T$ satisfying 1, 2, 3 exists then $T$ is a multiplicative LIM on $S$ and $S$ is ELA.

4. If $B$ in this theorem is only a locally convex linear topological space then, under the same conditions, there exists a linear operator $T$ for which 1 and 3 hold.

III. The support functional for the set of LIM's and left almost convergence. Let $S$ be a semigroup having the finite intersection property for right ideals (f.i.p.r.i.) i.e. such that $\bigcap_{1}^{n} s_{i} S \neq \varnothing$ for any finite subset $\left\{s_{1}, \ldots, s_{n}\right\} \subset S$. Then the (right) partial order defined by $b \geqq a$ iff $b \in a S \cup\{a\}$ renders $S$ a directed set (see introduction). We shall deal in what follows only with this (right) partial order, even when $S$ will be amenable. In the whole of this section

$$
\lim \sup _{s} f(s)=\inf _{s}\left[\sup _{t \geqq s} f(t)\right], \quad \liminf _{s} f(s)=\sup _{s}\left[\inf _{t \geqq s} f(t)\right] \text { and } \lim _{s} f(s)
$$

are defined with respect to this right partial order.

Denote by $Q(f)=\lim \sup _{s} f(s)$. Then $Q(f+g) \leqq Q(f)+Q(g), Q(\lambda f)=\lambda Q(f)$ if $\lambda \geqq 0$ and $-Q(-f)=\lim \inf _{s} f(s)$. Furthermore $Q(f)=\inf _{s \in S}\left[\sup _{t \in S} f(s t)\right.$ ]. Since $\sup _{u \geqq s} f(u) \geqq \sup _{t} f(s t),(u \geqq s$ iff $u \in s S \cup\{s\})$, one has $Q(f) \geqq \inf _{s} \sup _{t} f(s t)$. But if $t_{0} \in S$ then $\sup _{u \geqq s t_{0}} f(u) \leqq \sup _{t} f(s t)$. Hence $Q(f) \leqq \sup _{t} f(s t)$ for any $s \in S$ thus 
$Q(f)=\inf _{s} \sup _{t} f(s t)$. Moreover $Q\left(f_{a}\right)=Q(f)$ for any $a \in S$. Clearly $Q\left(f_{a}\right)=$ $\inf _{s} \sup _{t} f(a s t) \geqq Q(f)$. But if $s \in S$ then $\sup _{t} f(s t) \geqq \sup _{t} f(u t)$ for any $u \in s S \cap a S$. Hence $\sup _{t} f(s t) \geqq \inf _{v} \sup _{t} f($ avt $)=Q\left(f_{a}\right)$. Hence $Q(f)=Q\left(f_{a}\right)$. We have thus proved the

Proposition 1. Let $S$ have the f.i.p.r.i. and let $Q(f)=\lim \sup _{s} f(s)$ for $f \in m(S)$. Then $Q(f)=\inf _{s} \sup _{t} f(s t), \quad Q(\lambda f)=\lambda Q(f)$ if $\lambda \geqq 0, \quad Q(f+g) \leqq Q(f)+Q(g)$ and $Q\left(f_{a}\right)=Q(f)$ for any $a \in S$ and $f, g \in m(S)$.

Remark. We did not assume that $S$ is LA or ELA but only that $S$ has the f.i.p.r.i. For example any group $S$ has this property and in this case

$$
Q(f)=\sup _{s \in s} f(s) .
$$

Denote by $M \subset m(S)^{*}$ the set of means and by $M_{r}=\{\phi \in M ; \phi(s S)=1$ for each $s$ in $S\}$ (i.e. the set of means which "live" on right ideals). Then $M_{r}$ is convex and $w^{*}$-compact. We show in the next proposition that $Q$ is the "support functional" of the convex set $M_{r}$.

Proposition 2. Let $S$ have the f.i.p.r.i. Then $Q(f)=\sup _{\phi \in M_{\tau}} \phi(f)$. Consequently a linear functional $\psi$ on $m(S)$ satisfies $\psi(f) \leqq Q(f)$ for all $f \in m(S)$ if and only if $\psi \in M_{r}$.

Proof. Let $\phi \in M_{r}$ and assume that $f, g \in m(S)$ are such that $f(t)=g(t)$ for $t \in s_{0} S$. Then $|\phi(f-g)| \leqq \phi(|f-g|)=\phi\left(|f-g| 1_{A}\right) \leqq\|f-g\| \phi(A)=0$, where $A$ is the complement of $s_{0} S$ in $S$. Thus $\phi(f)=\phi(g)$ if $f=g$ on some $s_{0} S$. If $f \in m(S)$ let $s_{0} \in S$ and define $f_{1} \in m(S)$ by $f_{1}(t)=f(t)$ for $t \in s_{0} S$ and $f_{1}(s)=\sup _{t} f\left(s_{0} t\right)$ if $s \notin s_{0} S$. Then $\phi(f)=\phi\left(f_{1}\right) \leqq \sup _{s} f_{1}(s)=\sup _{t} f\left(s_{0} t\right)$. Thus $\phi(f) \leqq \inf _{s} \sup _{t} f(s t)=Q(f)$ and so $\sup _{\phi \in M_{r}} \phi(f) \leqq Q(f)$. Let now $f_{0} \in m(S)$ be fixed and $Q\left(f_{0}\right)=\alpha$. Then there is a linear functional $\psi$ on $m(S)$ such that $\psi(f) \leqq Q(f)$ for $f \in m(S)$ and $\psi\left(f_{0}\right)=Q\left(f_{0}\right)$, by the Hahn-Banach theorem. But $\psi$ necessarily will satisfy $-Q(-f) \leqq \psi(f)$ $\leqq Q(f)$ for any $f \in m(S)$. If $f \geqq 0$ then $-Q(-f)=\lim \inf _{s} f(s) \geqq 0$ and $Q(1)=$ $-Q(-1)=1$. This implies that $\psi$ is a mean. Let $a \in S$. Then $\inf _{t} 1_{a s}(a t)=1$. Thus $\sup _{s} \inf _{t} 1_{a S}(s t)=1=-Q\left(-1_{a S}\right)$. Thus $\psi(a S) \geqq 1$. Since $\psi$ is a mean and $\psi(a S)=1$ for any $a \in S, \psi \in M_{r}$ and $\psi\left(f_{0}\right)=Q\left(f_{0}\right)$. Thus $\sup _{\psi \in M_{r}} \psi\left(f_{0}\right) \geqq Q\left(f_{0}\right)$. Since any linear $\psi$ on $m(S)$ dominated by $Q$ is necessarily a mean (and hence in $\left.m(S)^{*}\right)$ and by Corollary 2 on p. 22 of [2] we get this proposition.

Remark. Let $S$ have the f.i.p.r.i. then any extreme point $\phi$ of $M_{r}$ is an extreme point of the set of means $M$ (i.e. multiplicative). Since if $\phi=\frac{1}{2}\left(\phi_{1}+\phi_{2}\right)$ with $\phi_{1}, \phi_{2} \in M$ then $\phi_{1}(a S)+\phi_{2}(a S)=2$ and $\phi_{i}(a S) \leqq 1$. Hence $\phi_{1}(a S)=\phi_{2}(a S)=1$ for $a \in S$. Thus $\phi_{1}, \phi_{2} \in M_{r}$ and $\phi_{1}=\phi_{2}$. In fact the set of extreme points of $M_{r}$ coincides with the set $\bigcap_{s \in S}(s S)^{-}$, where bar denotes $w^{* *}$-closure in $\beta S$. (This intersection is not empty since $\bigcap_{i=1}^{n} s_{i} S \neq \varnothing$ for $\left\{s_{1}, \ldots, s_{n}\right\} \subset S$.) Semigroups with the f.i.p.r.i. and in particular the set $\bigcap_{s \in S}(s S)^{-}$have been considered in more detail by J. Sorenson in [25]. The above expression for $Q(f)$ does though not appear there. 
We get as a corollary the following characterization of ELA semigroups:

Corollary 3. Let $S$ be a semigroup.

(a) If $S$ is ELA then $\lim \sup _{s}\left(f-f_{a}\right)(s)=\lim \inf _{s}\left(f-f_{a}\right)(s)=0$ for any $f \in m(S)$ and $a \in S$.

(b) If $S$ has the f.i.p.r.i. and lim $\sup _{s}\left(f-f_{a}\right)(s) \leqq 0$ for each $f \in m(S)$ and $a \in S$ then $S$ is ELA.

Proof (a). If $S$ is ELA and $a \in S$ let $b \in S$ satisfy $a b=b$. If $c \geqq b$ then $c=b$ or $c=b c^{\prime}$ with $c^{\prime} \in S$. In both cases $f(c)-f(a c)=0$.

(b) If $S$ has the f.i.p.r.i. and $Q\left(f-f_{a}\right) \leqq 0$ for $f \in m(S)$ and $a \in S$ then let $\phi \in \bigcap_{s \in S}(s S)^{-}$(where bar denotes $w^{*}$-closure in $\beta S$ ). Then $\phi \in M_{r}$ and $\phi(f) \leqq Q(f)$ for all $f \in m(S)$. Thus $\phi\left(f-f_{a}\right) \leqq 0$ and $\phi\left((-f)-\left(-f_{a}\right)\right) \leqq 0$ i.e. $\phi\left(f-f_{a}\right)=0$ for all $a \in S$. Thus $\phi$ is a multiplicative LIM and $S$ is ELA.

CoRollary 4. If $S$ is ELA then the set of LIM's coincides with $M_{r}$ and consequently the support functional of the set of LIM's is

$$
Q(f)=\inf _{s} \sup _{t} f(s t)=\limsup _{s} f(s)=\sup _{\phi \in M_{r}} \phi(f) .
$$

Proof. From Lemma 1 of $\$ 2, M_{r}$ and the set of LIM's have the same extreme points and are both $w^{*}$-compact and convex. By the Krein-Mil'man theorem they coincide. $Q(f)$ being the support functional of $M_{r}$ is also the support functional of the set of LIM's.

REMARK. If $S$ is LA and $M_{r}$ coincides with the set of LIM's then $S$ is ELA (since $\varnothing \neq \bigcap_{s \in S}(s S)^{-} \subset M_{r} \cap \beta S$ ).

Lemma 5. If $S$ is ELA then $H=K$ and

$$
\inf _{h \in H} \sup _{s}[f(s)+h(s)]=\inf _{s} \sup _{t} f(s t) \quad \text { for } f \in m(S) .
$$

Proof. That $H=K$ is immediate from Corollary 3 of $\S 2$.

Let $f \in m(S)$ be fixed. For any LIM $\phi$ and any $h \in H=K$ one has $\phi(f)=\phi(f+h)$ $\leqq \sup _{s}[f(s)+h(s)]$. Thus $\phi(f) \leqq \inf _{h \in H} \sup _{s}[f(s)+h(s)]$. Taking sup over $\phi$ in the set of LIM's one gets $Q(f) \leqq \inf _{h \in H} \sup _{s \in S}[f(s)+h(s)]$. Let now $a \in S$ and define $h \in H$ by $h(a S)=0$ and $h(s)=-f(s)+\sup _{t \in S} f(a t)$ if $s \notin a S$. Then $h=h-h_{a} \in H$

$$
\sup _{s \in S}[f(s)+h(s)] \leqq \sup _{t \in S} f(a t) .
$$

Hence $\inf _{h \in H} \sup _{s \in S}[f(s)+h(s)] \leqq \sup _{t \in S} f(a t)$ for any $a \in S$ which by Corollary 4 implies this lemma.

REMARKS. 1. It has been shown by Følner in [10, pp. 5-6] that if $G$ is an amenable group then

$$
\inf _{h \in H} \sup _{s \in G}[f(s)+h(s)]=\inf _{\mathscr{A}} \sup _{s \in G} \sum_{1}^{N} \alpha_{n} f\left(a_{n} s\right)
$$


where

$$
\mathscr{A}=\left\{\left(\alpha_{1}, \ldots, \alpha_{N} ; a_{1}, \ldots, a_{N}\right), \alpha_{n} \geqq 0, \sum_{1}^{N} \alpha_{n}=1, a_{n} \in G, N=1,2, \ldots\right\}
$$

and this common value is the support functional for the set of LIM's. He uses in his proof his deep characterization of amenable groups (see [9]). The above lemma could also be proved by first generalizing Følner's result in [10] to semigroups, using the result of Namioka in [22] and then showing that for ELA semigroups $S$ one has $\inf _{s} \sup _{t} f(s t)=\inf _{,} \sup _{t} \sum_{1}^{N} \alpha_{n} f\left(a_{n} t\right)$. This equality is readily seen: for any $\left(\alpha_{1}, \ldots, \alpha_{N}, a_{1}, \ldots, a_{N}\right)$ let $s_{0} \in S$ satisfy $a_{i} s_{0}=s_{0}$ for $1 \leqq i \leqq N$. Then

$$
\sup _{t} \sum \alpha_{n} f\left(a_{n} t\right) \geqq \sup _{t} \sum \alpha_{n} f\left(a_{n} s_{0} t\right)=\sup _{t} f\left(s_{0} t\right) \geqq \inf _{s} \sup _{t} f(s t)
$$

which readily implies the above equality.

2. Let $Z$ denote the additive group of integers and let $A \subset m(Z)$ be the subalgebra of $x=\{x(n)\} \in m(Z)$ for which $\phi(x)=\lim _{n \rightarrow+\infty} x(n)$ exists. Then $\phi$ is a multiplicative translation invariant mean on $A$ i.e. $A$ is ELA. For $x \in A$ let $p(x)=\inf _{n \in Z} \sup _{k \in Z} x(n+k)=\sup _{n \in Z} x(n)$. Then $p(x)$ is not the support functional of the set of LIM's on $A$. Since $\psi(x)=x(0)$ for $x \in A$ is dominated by $p$ but is not translation invariant. This shows that one should be careful when trying to generalize the above results to ELA subalgebras of $m(S)$ if $S$ is not ELA (see [8, p. 14]). Summarizing part of the above results and denoting by $K(f)$ the set of reals $c$ for which $c \cdot 1$ is in the pointwise (or equivalently the $w^{*}$ ) closure of $\left\{r_{s} f ; s \in S\right\}$. One has

THEOREM 6. Let $S$ be ELA and $Q(f)=\sup \{\phi(f) ; \phi \in L I M\}$. Then

$$
\begin{aligned}
Q(f) & =\limsup _{s} f(s)=\inf _{s} \sup _{t} f(s t)=\inf _{/ d} \sup _{s}\left[\sum_{1}^{n} \alpha_{i} f\left(a_{i} s\right)\right] \\
& =\inf _{h \in H} \sup _{s}[f(s)+h(s)]=\sup \{c ; c \in K(f)\} .
\end{aligned}
$$

Furthermore $f \in m(S)$ is left almost convergent to $\alpha$ if and only if $\lim _{s} f(s)$ exists and equals $\alpha$ or equivalently if and only if $K(f)=\{\alpha\}$. (Compare with Mitchell [21, p. 253].)

Proof. $Q(f)=\sup \phi(f)$ where the sup is over the set of multiplicative LIM's. Now by 2 of Theorem $A$ in the introduction of [16] one gets that $\sup \{c ; c \in K(f)\}$ $=Q(f)$. The other equalities for $Q(f)$ have been shown above.

By the Hahn-Banach theorem, $f_{0} \in m(S)$ is left almost convergent to $\alpha$ if and only if $-Q\left(-f_{0}\right)=Q\left(f_{0}\right)=\alpha$ i.e. if and only if $\lim \inf _{s} f(s)=\lim \sup _{s} f(s)=\alpha$. This is equivalent to $K(f)=\{\alpha\}$ again by 2 of Theorem $\mathrm{A}$ in [16].

EXAMPLE. Let $S$ be an EA semigroup. Denote by $Q_{L}\left(Q_{R}\right)$ the support functional of the set of LIM's (RIM's). Then $Q_{L}(f)=\inf _{s} \sup _{t} f(s t)$ and $Q_{R}(f)=\inf _{t} \sup _{s} f(s t)$. We give in what follows an example of a semigroup which is EA for which $Q_{L} \neq Q_{R}$ i.e. for which there is a multiplicative LIM which is not a RIM (and a multiplicative 
RIM which is not a LIM). In the case of amenable groups such an example is given in Hewitt-Ross [18, p. 239].

Let $G$ be the free group on the set of generators $\left\{t_{\alpha} ; \alpha \in I\right\}$ where $I$ is the set of ordinals less than the first incountable one (and $\geqq 1$ ). For $\alpha \in I, \alpha \geqq 2$ let $G_{\alpha}$ be the free subgroup generated by $\left\{t_{\beta} ; 1 \leqq \beta<\alpha\right\}$. One has $t_{\alpha}^{-1} G_{\alpha} t_{\alpha} \cap G_{\alpha}=\{e\}$ for each $\alpha \geqq 2$. Since if $t_{\alpha}^{-1} g t_{\alpha}=g^{\prime}$ for $g, g^{\prime}$ in $G_{\alpha}$ and $g^{\prime} \neq e$ (the identity of $G$ ) then $g \neq e$. Thus $g, g^{\prime}$ can be written in reduced form as $g=t_{\alpha_{1}}^{n_{1}} \cdots t_{\alpha_{j}}^{n_{j}}, g^{\prime}=t_{\beta_{1}}^{m_{1}} \cdots t_{\beta_{k}}^{m_{k}}$ where $n_{i} \neq 0, m_{i} \neq 0$ are integers $\alpha_{n}<\alpha, \beta_{m}<\alpha$ for $1 \leqq n<j, 1 \leqq m<k$ and the $\alpha_{n}$ 's are different and the $\beta_{m}$ 's are different. But then $t_{\alpha}^{-1} g t_{\alpha}=t_{\alpha}^{-1} t_{\alpha_{1}}^{n_{1}} \cdots t_{\alpha_{j}}^{n_{j}} t_{\alpha}=t_{\beta_{1}}^{m_{1}} \cdots t_{\beta k}^{m_{k}}$ where both sides are given in reduced form and $\beta_{m}<\alpha$ for $1 \leqq m \leqq k$. This cannot be since $G$ is a free group. Denote now $s=t_{1}$. Then $G_{\alpha} t_{\alpha} \cap \cap G_{\alpha} t_{\alpha}=\varnothing$ for any $\alpha \geqq 2$. Since if $G_{\alpha} t_{\alpha} s=G_{\alpha} t_{\alpha}$ for some $\alpha \geqq 2$ then $s \in t_{\alpha}^{-1} G_{\alpha} t_{\alpha}$ and $s \in G_{2} \subset G_{\alpha}$, thus $s=t_{1}=e$, which cannot be.

Let now (S) be the semigroup consisting of all countable subsets of $G$ with usual multiplication of subsets. Consider the net of point measures $\left\{1_{G_{\alpha} t_{\alpha}} ; \alpha \in I\right\} \subset m(\mathbb{S})^{*}$. If $A \in$ SS then $A \subset G_{\alpha_{0}}$ for some $\alpha_{0}$ and so $A G_{\alpha} t_{\alpha}=G_{\alpha} t_{\alpha}$ if $\alpha \geqq \alpha_{0}$. Thus

$$
\lim _{\alpha}\left\|L_{A} 1_{G_{\alpha} t_{\alpha}}-1_{G_{\alpha} t_{\alpha}}\right\|=\lim _{\alpha}\left\|1_{A G_{\alpha} t_{\alpha}}-1_{G_{\alpha} t_{\alpha}}\right\|=0
$$

(the norms being in $\left.m(\mathfrak{S})^{*}\right)$. Therefore if $\phi \in \beta(\mathfrak{S})$ is any $w^{*}$-limit of a subnet $\left\{1_{G_{v} t_{v}}\right\}$ of $\left\{1_{G_{\alpha} t_{\alpha}}\right\}$ then $\phi$ is a multiplicative LIM on $m(\mathscr{B})$. We claim that $\phi$ cannot be right invariant. If $\phi$ would be right invariant then by Lemma 1 in $\S 2$,

$$
\lim _{v}\left\|R_{A} 1_{G_{v} t_{v}}-1_{G_{v} t_{v}}\right\|=\lim _{v}\left\|1_{G_{v} t_{v} A}-1_{G_{v} t_{v}}\right\|=0
$$

for any $A \in$ SS. But take $A=\{s\}$. Then $G_{v} t_{v} A \neq G_{v} t_{v}$ for any $\nu$. Thus $\left\|1_{G_{v} t_{v} A}-1_{G_{v} t_{v}}\right\|=2$ for each $\nu$ and so $\phi$ is not right invariant. Thus $Q_{L} \neq Q_{R}$. If we denote by $H=H_{l}$ and $H_{r}$ all functions in $m(\mathbb{B S})$ representable $\sum_{1}^{n} f_{j}\left(g_{j}-r_{A_{j}} g_{j}\right)$ where $A_{j} \in$ BS are arbitrary and $f_{j}, g_{j} \in m(\mathbb{S})$ then we shall have for the semigroup \&S that $\bar{H}_{l} \neq \bar{H}_{r}$. Since

$$
Q_{L}(f)=\inf _{h \in H_{l}}\left[\sup _{s} f(s)+h(s)\right]=\inf _{h \in H_{l}} \sup _{s}[f(s)+h(s)]
$$

and in analogy for $Q_{R}(f)$. Since $Q_{L} \neq Q_{R}$ one has $\bar{H}_{l} \neq \bar{H}_{r}$. Furthermore $H_{l}$ and $H_{r}$ are not dense in $m(\mathscr{S})$.

We bring now some results on left almost convergence.

Definition. For $\phi \in m(S)^{*}$ define $T_{\phi}: m(S) \rightarrow m(S)$ by $\left(T_{\phi} f\right)(s)=\phi\left(r_{s} f\right)=\phi\left(f^{s}\right)$. $T_{\phi}$ is linear and $\left\|T_{\phi} f\right\|<\|\phi\|\|f\|$. If $\phi$ is a finite mean, i.e. if $\phi=\sum_{1}^{n} \alpha_{i} 1_{s_{i}}, \alpha_{i} \geqq 0$, $\sum \alpha_{i}=1$ and $1_{s_{i}} \in m(S)^{*}$ are point measures, then $\left(T_{\phi} f\right)(x)=\sum_{1}^{n} \alpha_{i} f\left(a_{i} x\right)=\sum \alpha_{i} f_{a_{i}}(x)$. Thus, if $\operatorname{LO}(f)=\left\{f_{s} ; s \in S\right\}, \operatorname{RO}(f)=\left\{f^{s} ; s \in S\right\}$ and Co denotes convex hull, then $T_{\phi} f \in \mathrm{Co} \operatorname{LO}(f)$ for any finite mean $\phi$. The following proposition is partly due to M. Day [4, p. 539] for the case that $S$ is amenable, (where use of Eberlein's ergodic theorem is made in its proof) and partly due to K. Witz [27, p. 694]. It is a generalization of a theorem of G. G. Lorentz and is given here in slightly different form with straightforward proof. Denote by $C$ the constant functions of $m(S)$. 
TheOREM 7. Let $S$ be a LA semigroup. Then $C \oplus \bar{K}$ is the space of left almost convergent (l.a.c.) functions of $m(S)$, f being l.a.c. to $c$ if and only if $f \in \mathrm{Cl}+\bar{K}$ (thus $\bar{K}=\{f \in m(S) ; f$ is l.a.c. to zero $\})$. Furthermore:

1. If $f$ is l.a.c. to $c$ and $\left\{\phi_{\alpha}\right\}$ is any net of means such that $\lim _{\alpha}\left\|L_{s} \phi_{\alpha}-\phi_{\alpha}\right\|=0$ for each $s \in S$ then $\lim _{\alpha}\left\|T_{\phi_{\alpha}} f-c 1\right\|=0$.

2. If $c 1$ is in the uniform closure of $\mathrm{Co} L O(f)$ then $f$ is l.a.c. to $c$.

REMARK 1. One cannot replace " $S$ is amenable" by " $S$ is LA" in Eberlein's ergodic theorem as stated in Day [4, pp. 536-537]. The example given in the remark after Theorem 6 in $\$ 2$ would violate (c) on p. 537 (see also p. 538) of [4].

Proof. Let $f$ be left almost convergent (l.a.c.) to zero. Then $f \in \bar{K}$. Otherwise there would be some $\phi \in m(S)^{*}$ with $\phi(\bar{K})=0$ and $\phi(f) \neq 0$, by the Hahn-Banach theorem. But then $\phi$ is left invariant and hence $\phi=\alpha \phi_{1}-\beta \phi_{2}$ where $\phi_{1}, \phi_{2}$ are LIM's and $\alpha, \beta \geqq 0\left[13\right.$, p. 55]. But then $\phi_{1}(f)=\phi_{2}(f)=0$ i.e. $\phi(f)=0$ which cannot be. Thus $f \in \bar{K}$. Since $\phi(\bar{K})=0$ for any $\operatorname{LIM} \phi$ we get that $\bar{K}$ coincides with the space of $f \in m(S)$ which are l.a.c. to zero. If $f$ is l.a.c. to $c$ then $f-c 1$ is l.a.c. to zero so $f \in c 1+\bar{K}$. Any $f \in c 1+\bar{K}$ is obviously l.a.c. to $c$. If $c 1 \in \bar{K}$ and $\phi$ is a LIM then $c=\phi(c 1)=0$. Thus $C+\bar{K}$ is a direct sum ( $1 \in \bar{K}$ for nonamenable groups $S$ !). That 2 holds is immediate: If $\phi$ is a LIM then $\phi(f)=\phi(g)$ for any $g \in \operatorname{Co} \operatorname{LO}(f)$ and since $c 1$ can be uniformly approximated by such $g$ 's one has $c=\phi(c 1)=\phi(f)$ for any LIM $\phi$. We show now 1: Let $f$ be l.a.c. to $c$ and $\left\{\phi_{\alpha}\right\}$ be a net of means with $\left\|L_{s} \phi_{\alpha}-\phi_{\alpha}\right\| \rightarrow 0$ for $s \in S$. If $g \in m(S)$ and $a \in S$ then

$$
\begin{aligned}
\left|T_{\phi_{a}}\left(g-g_{a}\right)(x)\right| & =\left|\phi_{\alpha}\left(r_{x} g-r_{x} l_{a} g\right)\right|=\left|\phi_{\alpha}\left(r_{x} g-l_{a} r_{x} g\right)\right|=\left|\phi_{\alpha} g^{x}-\left(L_{a} \phi_{\alpha}\right) g^{x}\right| \\
& =\left|\left(\phi_{\alpha}-\left(L_{a} \phi_{\alpha}\right) g^{x}\right)\right| \leqq\left\|L_{a} \phi_{\alpha}-\phi_{\alpha}\right\|\|g\| \rightarrow 0 .
\end{aligned}
$$

Thus $\left\|T_{\phi_{a}}\left(g-g_{a}\right)\right\| \rightarrow 0$ for each $g \in m(S)$ and $a \in S$. Now any $g \in K$ is given as $g=\sum_{1}^{n}\left(g_{i}-r_{a_{i}} g_{i}\right)$ thus $\left\|T_{\phi_{a}} g\right\| \rightarrow 0$ for each $g \in K$. If $g_{0} \in \bar{K}$ let $g \in K$ be such that $\left\|g-g_{0}\right\|<\varepsilon$. Then $\left\|T_{\phi_{\alpha}} g_{0}\right\| \leqq\left\|T_{\phi_{\alpha}}\left(g_{0}-g\right)\right\|+\left\|T_{\phi_{\alpha}} g\right\| \leqq\left\|\phi_{\alpha}\right\| \varepsilon+\left\|T_{\phi_{\alpha}} g\right\|=\varepsilon+\left\|T_{\phi_{\alpha}} g\right\|$ $\rightarrow \varepsilon$. Thus $\left\|T_{\phi_{\alpha}} g_{0}\right\| \rightarrow 0$ for any $g_{0} \in \bar{K}$. If $f$ is l.a.c. to $c$ then $f=c 1+g_{0}$ for some $g_{0} \in \bar{K}$. Thus $T_{\phi_{\alpha}} f=c\left(T_{\phi_{\alpha}} 1\right)+T_{\phi_{\alpha}} g_{0}=c 1+T_{\phi_{\alpha}} g_{0} \rightarrow c 1$ uniformly on $S$ which finishes the proof.

Remark. Define $P$ : $C \oplus \bar{K} \rightarrow C \oplus \bar{K}$ by: $P f=\phi(f) .1$ where $\phi$ is any LIM and $1 \in m(S)$ (i.e. the constant $\phi(f)$ valued function). Then $P^{2}=P$ and by Theorem 7 , $\lim _{\alpha}\left\|\left(T_{\phi_{\alpha}}-P\right) f\right\|=0$ for all $f \in C \oplus \bar{K}$. It is though not true in general that $\left\|T_{\phi_{\alpha}}-P\right\| \rightarrow 0$ in the uniform operator norm over $C \oplus \bar{K}$, even for ELA semigroups $S$. In fact let $S=\{1,2, \ldots\}$ with $s t=\max (s, t)=s \vee t$ let $\phi_{n}=1_{n} \in m(S)^{*}$. Then $\lim _{n \rightarrow \infty}\left\|L_{s} \phi_{n}-\phi_{n}\right\|=0$ for all $s \in S$ since $L_{s} \phi_{n}=\phi_{s \cdot n}=\phi_{n}$ if $n \geqq s$. If $\left\|T_{\phi_{n}}-P\right\|$ $\rightarrow 0$ then since $P(\bar{K})=0$ one would have that $\left\|T_{\phi_{n}} h\right\| \rightarrow 0$ uniformly in $h \in \bar{K}$ with $\|h\| \leqq 2$, i.e. $\left\|T_{\phi_{n}}\left(f-f_{s}\right)\right\| \rightarrow 0$ uniformly both for $s \in S$ and $f \in m(S)$ with $\|f\| \leqq 1$. But $\left[T_{\phi_{n}}\left(f-f_{s}\right)\right](t)=-\left(L_{s} \phi_{n}-\phi_{n}\right)\left(f^{t}\right)$ so $\left\|T_{\phi_{n}}\left(f-f_{s}\right)\right\| \geqq\left|\left(L_{s} \phi_{n}-\phi_{n}\right) f^{t}\right|$ for any $t$ and thus for $t=1$. Thus $\left\|T_{\phi_{n}}\left(f-f_{s}\right)\right\| \geqq\left|\left(L_{s} \phi_{n}-\phi_{n}\right) f\right|$ for all $f \in m(S)$ with $\|f\| \leqq 1$, and all $s \in S$. Hence $\left\|L_{s} \phi_{n}-\phi_{n}\right\| \rightarrow 0$ uniformly for $s \in S$. But $\left\|L_{s} \phi_{n}-\phi_{n}\right\|=0$ or 2 . If $n_{0} \in S$ is such that $n \geqq n_{0}$ implies $\left\|L_{s} \phi_{n}-\phi_{n}\right\|<2$ for all $s \in S$ then $L_{s} \phi_{n}=\phi_{n}$ for 
all $s \in S$ and $n \geqq n_{0}$. But $L_{s} \phi_{n_{0}}=1_{s \cdot n_{0}}=1_{s}$ if $s=n_{0}+1$. Thus $\phi_{n_{0}+1}=\phi_{n_{0}}$ which is false. This example shows that a "uniform ergodic theorem" does not generally hold even for abelian EA semigroups.

THEOREM 8. Let $S$ be ELA. Then $C \oplus \bar{H},(\bar{H})$, is the space of $f \in m(S)$ which is l.a.c. (to zero). Moreover the following are equivalent: (1) $f$ is l.a.c. to c. (2) $f \in c 1+\bar{H}$. (3) $c 1$ is in the uniform closure of $L O(f)$. (4) $\lim _{s} f(s)$ exists and equals $c$. (5) The $w^{*}$ (or pointwise) closure of $R O(f)=\left\{r_{s} f ; s \in S\right\}$ contains a unique constant function which is $c 1$.

If $A \subset S$ and $1_{A}$ is 1.a.c. then $1_{A}$ is 1.a.c. to 0 or 1 . Moreover $1_{A}$ is l.a.c. to $0 \Leftrightarrow A \cap a S=\varnothing$ for some $a \in S$ and $1_{A}$ is 1.a.c. to $1 \Leftrightarrow a S \subset A$ for some $a \in S$. In fact $\lim _{s} 1_{A}(s)=0$ or 1 and $Q\left(1_{A}\right)=\inf _{s} \sup _{t} 1_{A}(s t)=0 \Leftrightarrow \sup 1_{A}(a t)=0$ for some $a \in S$ i.e. $a S \cap A=\varnothing$ for some $a \in S$.

Proof. Let $\phi$ be a multiplicative LIM and $1_{s_{\alpha}} \rightarrow \phi$ in $w^{*}$. Then $\left\|L_{s} 1_{s_{\alpha}}-1_{s_{\alpha}}\right\| \rightarrow 0$ for each $s \in S$ by Lemma 1 of $\S 2$. Let $\phi_{\alpha}=1_{s_{\alpha}}$. If $f$ is l.a.c. to $c$ then $T_{\phi_{\alpha}} f=f_{s_{\alpha}} \rightarrow c 1$ uniformly by 1 of the previous proposition. Theorems 6,7 imply the equivalences (1)-(5).

REMARKS. Consider again the semigroup given after Theorem 6 . We have shown there that $\bar{H}_{l} \neq \bar{H}_{r}$ and $H_{r}, H_{l}$ are not dense in $m($ (S) $)$. If $f \in \bar{H}_{l}$ and $f \notin \bar{H}_{r}$ then $f$ is l.a.c. to zero but $f$ is not right almost convergent at all. Since if $f$ would be right almost convergent to $c$ let $\phi$ be a left and right invariant mean. Then $\phi(f)=0$ i.e. $c=0$ and so $f \in \bar{H}_{r}$ which cannot be. Thus there exist two multiplicative right invariant means $\phi_{1}, \phi_{2}$ with $\alpha=\phi_{1}(f) \neq \phi_{2}(f)=\beta$. Thus by 2 of Theorem $\mathrm{A}$ in the introduction of [16] the functions $\alpha 1$ and $\beta 1$ are in the pointwise closure (which is the same as the $w^{*}$ closure) of $\operatorname{LO}(f)$. While the only constant function which is in the uniform closure of $\operatorname{LO}(f)$ (or even of $\mathrm{Co} \operatorname{LO}(f)$ ) is the constant zero function. Use of Mitchell's Theorem 4 in [21, p. 253] and the fact that $[\alpha, \beta] \subset\{\psi(f) ; \psi \in \mathbf{R I M}\}$ implies even that for any $\alpha<\gamma<\beta, \gamma 1$ belongs to the pointwise (or $w^{*}$ ) closure of Co $\operatorname{LO}(f)$. As to the right orbit of $f, \operatorname{RO}(f)$, its $w^{*}$-closure contains a unique constant function which is none other than 0 . The zero function and for that matter any other constant function, is though not in the uniform closure of $\operatorname{Co} \operatorname{RO}(f)$ since then $f$ would be right almost convergent.

Some more examples of ELA semigroups. 1 . Let $G$ be a group. Denote by $G_{c}$ the semigroup of all finite or countable subsets of $G$ with the usual multiplication $A B=\{a b ; a \in A, b \in B\}$. Then $G_{c}$ is an EA semigroup (in which $G$ is embedded). Since if $A, B \in G_{c}$ and $C$ is the group generated by $A \cup B$ then $C A=C B=A C$ $=B C=C$. If $G$ is not countable then $G_{c}$ does not contain a zero. If $G, G^{\prime}$ are groups with identities $e, e^{\prime}$ (resp.) and if $\phi: G_{c} \rightarrow G_{c}^{\prime}$ is an isomorphism then for any $g \in G$ $\left\{e^{\prime}\right\}=\phi(\{e\})=\phi(\{g\}) \phi\left(\left\{g^{-1}\right\}\right)$. Hence $\phi(\{g\}) \subset G$ contains just one element of $G^{\prime}$. Thus $\phi$ maps $(\{g\} ; g \in G\}$ into $\left\{\left\{g^{\prime}\right\} ; g^{\prime} \in G^{\prime}\right\}$ and is one to one. This readily implies that $G$ and $G^{\prime}$ are isomorphic. Thus nonisomorphic groups $G$ give rise to nonisomorphic EA semigroups $G_{c}$. $G_{c}$ will not have left or right cancellation. 
2. If $G$ is any locally finite group (i.e. such that finite subsets generate finite subgroups), let $G_{f}$ be the semigroup of all finite subsets of $G$ with the same multiplication as above. Then $G_{f}$ is a EA semigroup (which does not have a zero if $G$ is not finite), in which the group $G$ is embedded. As above nonisomorphic locally finite groups $G$ give rise to nonisomorphic EA semigroups $G_{f}$. One can construct from any semigroup $S$ the semigroup $S_{f}$ and it is readily seen that if $S$ is ELA, ERA or EA then $S_{f}$ is ELA, ERA or EA resp. It may though happen that $S_{f}$ is EA without $S$ being so (as is the case if $S$ is any nontrivial locally finite group).

4. Let $S=\{(m, n) ; m, n=1,2, \ldots\}$ with the multiplication $\left(m_{1}, n_{1}\right)\left(m_{2}, n_{2}\right)$ $=\left(m_{2}, n_{2}\right)\left(n_{1}, m_{1}\right)=\left(m_{2}, n_{2}\right)$ if $m_{1}<m_{2}$ and $\left(m_{1}, n_{1}\right)\left(m_{1}, n_{2}\right)=\left(m_{1}, n_{1}+n_{2}\right) . S$ is an abelian EA semigroup with no idempotents. This example is taken from Ljapin $[19$, p. 69] (where it appears in different context). A slight generalization of this construction which yields a large class of EA semigroups is as follows: Let $I$ be an infinite set and $\left\{S_{\alpha} ; \alpha \in I\right\}$ a collection of semigroups. Linearly order $I$ so that it contains no last element. Let $S=\bigcup_{\alpha \in I} S_{\alpha}$ and denote the elements of $S$ as pairs $(\alpha, s)$ (or $(\beta, t)$ etc...) where $s \in S_{\alpha}$ and $\alpha$ ranges over $I$. Define $(\alpha, s)(\beta, t)=$ $(\beta, t)(\alpha, s)=(\beta, t)$ if $\alpha<\beta$ and $(\alpha, s)(\alpha, t)=(\alpha, s t) . S$ becomes this way an EA semigroup in which each $S_{\alpha}$ is embedded. $S$ will be commutative iff all $S_{\alpha}$ are. $S$, as any other nontrivial EA semigroup, will never have left or right cancellation.

5. Let $S$ be a semigroup generated by one element. Then $S$ is ELA if and only if $S$ is finite and has a zero. Since if $S=\left\{a^{n}\right\}^{\infty}$, is ELA, let $p>0$ be such that $a a^{p}$ $=a^{2} a^{p}=a^{p}$. Then $a^{n}=a^{p}$ if $n \geqq p$. Thus $S$ is a finite ELA semigroup with zero $a^{p}$. The multiplicative semigroup $\{0,2,4,8, \ldots\}$ is, EA, abelian infinite and is generated by the two elements $\{0,2\}$ (clearly any finitely generated ELA semigroup contains a right zero).

6 . Let $S=[1, \Omega)$ be the semigroup of ordinals less than the first incountable ordinal $\Omega$ with the usual (noncommutative) addition + of order types. Then $S$ is a left cancellation semigroup (see Kamke [29, p. 60 and p. 94]) which is ELA but is not right amenable. In fact if $\alpha \in S$ then $\alpha+(\alpha \omega+\xi)=\alpha(1+\omega)+\xi=\alpha \omega+\xi$ for any $\xi \in S$. (See Kamke [29, p. 64].) If $\alpha, \beta \in S$ and $\gamma>\max \{\alpha \omega, \beta \omega\}$ where $\gamma \in S$ then there are $\xi, \eta \in S$ such that $\gamma=\alpha \omega+\xi=\beta \omega+\eta$ (see Kamke [29, p. 94]). Thus $\alpha+\gamma$ $=\beta+\gamma=\gamma . S$ is not RA since $(S+\omega) \cap(S+1)=\varnothing . S$ contains no idempotents.

The examples given here together with those of [15], [16] should convince the reader that the class of ELA semigroups is indeed immense and worth being investigated in more detail.

\section{Added in Proof.}

1. R. R. Phelps has kindly informed the author that Theorem 4 in $\$ 2$ generalizes Theorem 1.1 of R. R. Phelps, Extreme positive operators and homomorphisms, Trans. Amer. Math. Soc. 108 (1963), 265-274, which is probably a more precise reference than the Bonsall-Lindenstrauss-Phelps paper quoted. Furthermore, unlike pointed out in the introduction, Theorem 4 of $\$ 2$ does have an analogue (with some restrictions, though) to the left amenable case. This analogue has been 
obtained by G. Converse, I. Namioka, and R. R. Phelps in a paper which was being prepared for publication after this paper had been sent for publication.

2. Concerning Theorem 6 of $\S 3$, the following remark is of interest:

It is known that for a LA semigroup $S$, a set $A \subset S$ has the property that $\phi(A)=1$ for some LIM $\phi$ on $m(S)$ if and only if the sets $\left\{s^{-1} A ; s \in S\right\}$ have the finite intersection property (where $s^{-1} A=\{t \in S$; st $\in A\}$ ), i.e. $A$ is left thick (see Mitchell [21, p. 257]). For an ELA semigroup $S$ we have the

Proposition. Let $S$ be ELA and $A \subset S$. Then there is some LIM $\phi$ on $m(S)$ such that $\phi(A)>0$ if and only if $s^{-1} A \neq \varnothing$ for all $s \in S$.

Proof. Since $l_{S} 1_{A}=1_{S}{ }^{-1} A$ the "only if" part is trivial. For the "if" part let $\left\{1_{s_{\alpha}}\right\} \subset m(S)^{*}$ be a net of point measures such that $1_{s_{\alpha}} \rightarrow \phi$ in $w^{*}$ where $\phi$ is a multiplicative LIM on $m(S)$. Then: $\left({ }^{*}\right)$ for any $s \in S$ there is some $\alpha_{0}$ with $s s_{\alpha}=s_{\alpha}$ if $\alpha \geqq \alpha_{0}$. Choose for each $\alpha, t_{\alpha} \in S$ such that $s_{\alpha} t_{\alpha} \in A$. If $u_{\alpha}=s_{\alpha} t_{\alpha}$ then $\left\{u_{\alpha}\right\}$ enjoys property $\left({ }^{*}\right)$ and $\left\{u_{\alpha}\right\} \subset A$. Thus $\lim \left\|L_{s} 1_{u_{\alpha}}-1_{u_{\alpha}}\right\|=0$ for all $s \in S$. Any $w^{*}$ cluster point $\psi$ of $\left\{1_{u_{\alpha}}\right\}$ will be a multiplicative LIM on $m(S)$ such that $\psi(A)=1$, since $1_{u_{\alpha}}(A)=1$ for all $\alpha$. In particular, if $A \subset S$ satisfies $\psi_{1}(A)>0$ for some LIM $\psi_{1}$ on $m(S)$ then there is some multiplicative $\operatorname{LIM} \psi_{2}$ on $m(S)$ such that $\psi_{2}(A)=1$.

\section{REFERENCES}

1. F. F. Bonsall, J. Lindenstrauss and R. R. Phelps, Extreme positive operators on algebras of functions, Math. Scand. 18 (1966), 161-182.

2. M. M. Day, Normed linear spaces, Springer, Berlin, 1958.

3. - Means for the bounded functions and ergodicity of the bounded representations, Trans. Amer. Math. Soc. 69 (1950), 276-291.

4. —_, Amenable semigroups, Illinois J. Math. 1 (1957), 509-544.

5. - Fixed point theorems for compact convex sets, Illinois J. Math. 5 (1961), 585-590.

6. J. Dixmier, Les moyennes invariantes dans les semigroupes et leurs applications, Acta Sci. Math. Szeged 12 (1950), 213-227.

7. N. Dunford and J. Schwartz (with the assistance of W. G. Bade and R. G. Bartle), Linear operators. I, Interscience, New York, 1958.

8. E. Følner, Generalization of a theorem of Bogoliouloff to topological abelian groups, Math. Scand. 2 (1954), 5-18.

9. _- On groups with full Banach mean value, Math. Scand. 3 (1955), 243-254.

10. - Note on groups with and without full Banach mean value, Math. Scand. 5 (1957), 5-11.

11. A. H. Frey, Jr., Studies in amenable semigroups, Thesis, Univ. of Washington, Seattle, 1960.

12. I. M. Gelfand, D. A. Raikov and G. Shilov, Commutative normed rings, Chelsea, New York, 1964.

13. E. Granirer, On amenable semigroups with a finite dimensional set of invariant means. I, II, Illinois J. Math. 7 (1963), 32-48; 49-58.

14. - A theorem on amenable semigroups, Trans. Amer. Math. Soc. 111 (1964), 367-379.

15. —_, Extremely amenable semigroups, Math. Scand. 17 (1965), 177-197.

16. ---, Extremely amenable semigroups. II, Math. Scand. (to appear). 
17. E. Granirer, On the range of an invariant mean, Trans. Amer. Math. Soc. 125 (1966), 384394.

18. E. Hewitt and K. Ross, Abstract harmonic analysis. I, Springer, Berlin, 1963.

19. E. S. Ljapin, Semigroups, Transl. Math. Monographs, Vol. 3, Amer. Math. Soc., Providence, R. I., 1963.

20. L. Loomis, Abstract harmonic analysis, Van Nostrand, Princeton, N. J., 1953.

21. T. Mitchell, Constant functions and left intariant means on semigroups, Trans. Amer. Math. Soc. 119 (1965), 244-261.

22. T. Mitchell, Fixed points and multiplicative left invariant means, Trans. Amer. Math. Soc. 122 (1966), 195-202.

23. I. Namioka, Folner's conditions for amenable semigroups, Math. Scand. 15 (1964), 18-28.

24. R. J. Silverman, Invariant means and cones with vector interiors, Trans. Amer. Math. Soc. 88 (1958), 75-79; 327-330.

25. J. Sorenson, Existence of measures that are invariant under a semigroup of transformations, Thesis, Purdue Univ., Lafayette, Ind., 1966.

26. C. Wilde and K. Witz, Invariant means and the Stone-Cech compactification, Pacific J. Math. 21 (1967), 577-586.

27. K. Witz, Applications of a compactification for bounded operator semigroups, Illinois J. Math. 8 (1964), 685-696.

28. J. L. Kelly, General topology, Van Nostrand, Princeton, N. J., 1955.

29. E. Kamke, Theory of sets, Dover, New York, 1950.

30. I. Glicksberg, On convex hulls of translates, Pacific J. Math. 13 (1963), 97-113.

UNIVERSITÉ DE MONTRÉAL, Montreal, Canada

The University of British Columbia, VANCouver, Canada 